\title{
Data uncertainty in virtual network embedding Robust optimization and protection levels
}

\author{
S. Coniglio · A. Koster · M. Tieves
}

Received: date / Accepted: date

\begin{abstract}
We address the Virtual Network Embedding problem (VNE) which, given a physical (substrate) network and a collection of virtual networks (VNs), calls for an embedding of the most profitable subset of VNs onto the physical substrate, subject to capacity constraints. In practical applications, node and link demands of the different VNs are, typically, uncertain and difficult to know a priori. To face this issue, we first model VNE as a chance-constrained Mixed-Integer Linear Program (MILP) where the uncertain demands are assumed to be random variables. We then propose a $\Gamma$-robust optimization approach to approximate the original chance-constrained formulation, capable of yielding solutions with a large profit that are feasible for almost all the possible realizations of the uncertain demands. To solve larger scale instances, for which the exact approach is computationally too demanding, we propose two MILP-based heuristics: a parametric one, which relies on a parameter setting chosen a priori, and an adaptive one, which does not. We conclude by reporting on extensive computational experiments where the different methods and approaches are compared.
\end{abstract}

Keywords network virtualization $\cdot$ data uncertainty $\cdot \Gamma$-robustness $\cdot$ integer programming $\cdot$ math heuristics

The paper is an extended version of [1]. In its extended form, is has not been published or accepted for publication elsewhere, nor it has been submitted to any other journal.

S. Coniglio, A. Koster, M. Tieves

Lehrstuhl II für Mathematik, RWTH Aachen University

Tel.: +49 (0)2418094995

Fax.: +49(0)241 8092136

\{coniglio, koster, tieves\}@math2.rwth-aachen.de

Present address of S. Coniglio (corresponding author):

Department of Mathematical Sciences, University of Southampton

Tel.: +44023 80593644

s.coniglio@soton.ac.uk 


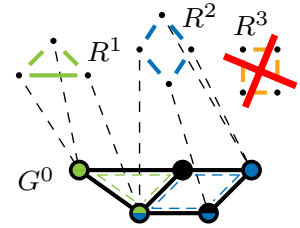

(a)

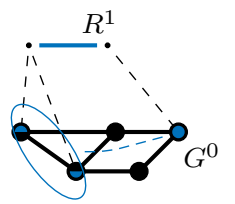

(b)

Fig. 1: (a) Two VN requests, $R^{1}$ and $R^{2}$, embedded into the substrate $G^{0}$, while $R^{3}$ is rejected. (b) A (forbidden) one-to-many mapping of a virtual node.

\section{Introduction}

In the context of large scale networks, the paradigm of network virtualization has garnered a large attention, being advocated as one of the key technologies of the future of networking $[2,3]$. In its general form, the paradigm allows to decouple the physical (low level) management aspects of a networking environment from those (of higher level) involving service provisioning. This way, it benefits the two main actors of a networking context: the owner of the physical or substrate network, the so-called infrastructure provider - who, this way, can solely concentrate on the management aspects of the substrate - and the service provider - who, this way, can only focus on the provisioning aspects of his services. A prominent application scenario is that of the Internet which, due to only allowing for small and incremental updates to its structure as a consequence of its inherently plural nature, can largely benefit from virtualization techniques as a noninvasive way of upgrading itself, preventing ossification phenomena. For a more detailed treatment of the topic, we refer the reader to the surveys $[2,3,4]$.

\subsection{The VNE problem and previous work}

When considering the infrastructure provider's perspective, we are faced with the so-called Virtual Network Embedding problem (VNE). It is the problem of, in the first place, deciding whether to accept or reject a subset of Virtual Network (VN) requests issued by the customers (the admission control aspect) and, then, of embedding the accepted VNs onto the substrate network, subject to capacity constraints. In this paper, we will focus on the offline version of $\mathrm{VNE}$ in which the set of VN requests is known beforehand. This suits the case where the $\mathrm{VN}$ requests are issued ahead of the time when their service will be activated, thus allowing for sufficient time for offline planning. Such requests are, typically, quite large in terms of resource requirements and, if accepted, sufficiently long lasting to assume that they will be embedded indefinitely.

In this work, we assume that each VN is composed of a set of virtual nodes, each of which endowed with an estimate of the node resources it requires (computing power), which we refer to as node demands, as well as a set of 
traffic or link demands between pairs of virtual nodes. An embedding thus consists of virtual-to-physical node-to-node and link-to-path mappings which do not exceed the node and link capacities of the substrate network. Note that, while we allow for the mapping of any number of virtual nodes belonging to the same VN request to the same physical node (so-called co-location), we forbid the mapping of a single virtual node to more than a single physical node ${ }^{1}$. As customary for VNE, see [3] and the references therein, we also consider locality constraints which restrict the set of physical nodes onto which a virtual node can be mapped. This allows for the encoding of technical specifications which are only met by certain physical nodes, as well as geographical restrictions (to prevent, for instance, the mapping of data intensive services too far away from the corresponding data centers). Throughout the paper, we will assume a single path unsplittable routing scheme, which is often preferred to a splittable one so to avoid packet reordering issues (see, e.g., [5]). Nevertheless, the generalization of the techniques that we will propose to the splittable case is straightforward, as we will better point out in the following. For an example of a VNE instance, together with a feasible solution, see Figure 1 (a).

As it is easy to observe, VNE is weakly $\mathcal{N} \mathcal{P}$-hard by reduction from the 0-1 knapsack problem (KP) [3]. Strong $\mathcal{N} \mathcal{P}$-hardness is established in [6] by reduction from the maximum stable set problem. The result also implies that VNE cannot be approximated in polynomial time within a factor of $\left|V^{0}\right|^{\frac{1}{2}-\epsilon}$ for any $\epsilon>0$, unless $\mathcal{P}=\mathcal{N} \mathcal{P}[6]$.

Most of the literature on VNE employs heuristics in which node and link mappings are carried out sequentially, usually in an online setting where the virtual network requests arrive over time. Examples can be found in, e.g., [7], which also accounts for reconfigurations of a given embedding, and [8], which employs both deterministic and randomized rounding techniques. For an extensive account on (mostly heuristic) algorithms for VNE, we refer the reader to the excellent survey [3]. For a rounding algorithm based on column generation, also encompassing admission control, see [9]. For a greedy algorithm based on the degree of utilization of the different physical nodes, see [10]. Also see [11] for an energy-efficient version of VNE subject to Gaussian traffic demands.

Among the few exact approaches, we mention that in [12], where the authors propose a Mixed-Integer Linear Programming (MILP) formulation to carry out each step of an algorithm for the online version of the problem, [13], which illustrates an MILP formulation for the offline version of VNE which also accounts for the installation (or rental) of network capacities (with a rent-at-bulk aspect), and $[14,15]$, which extend [12] to the energy-aware and fault-tolerant cases.

\footnotetext{
1 We assume that each task which can be parallelized on multiple physical nodes is described in a VN request via as many virtual nodes as the number of parallel threads it can use. This way, a task will be split only if, by splitting it, a more profitable embedding can be obtained. On the contrary, splitting a single virtual node would force us to split both the node and the traffic demands over the physical network in a not well defined way, see Figure 1 (b).
} 
For the closely related problem of Network Functions Virtualization (NFV), we refer the reader to [16], which addresses network planning of a mixed physical-virtual CDN (Content Delivery Network), tackled from a stochastic programming point of view, and to [17], for the embedding of network functions with congestion minimization.

\subsection{Contribution and outline of the work}

To our knowledge, almost all the works on VNE assume that node and link demands of the different $\mathrm{VNs}$ are known deterministically ${ }^{2}$. In a realistic setting though, it is reasonable to assume that, for each VN, the actual demands of computing power and bandwidth (i.e., node and traffic demands) entail significant uncertainty (e.g., because of measurement errors or variability over time).

To cope with this issue, in this work we propose a robust optimization formulation (based on the model of $\Gamma$-robustness) for VNE which, after selecting an appropriate value for the parameter $\Gamma$, allows to achieve solutions which (under mild assumptions) satisfy each of the constraints where uncertain parameters are involved with any desired probability. For clarity, we first introduce a chance-constrained formulation for VNE where node and link demands are assumed to be random variables and the constraints of the problem are required to be satisfied with high probability, and show how this formulation can be approximated via the $\Gamma$-robust formulation which is, computationally, more tractable.

The paper is organized as follows ${ }^{3}$. In Section 2, we illustrate a deterministic MILP formulation for VNE and discuss on the $\mathcal{N} \mathcal{P}$-hardness of its two natural subproblems. In Section 3, we describe the chance-constrained and MILP $\Gamma$-robust formulations. To tackle large-scale instances, we present, in Section 4, two MILP-based heuristics, both yielding $\Gamma$-robust solutions: a two-phase one, which relies on an a priori parameter setting, and an adaptive one which does not. Extensive computational experiments are reported and illustrated in Section 5. Concluding remarks are drawn in Section 6.

\section{Deterministic MILP formulation and complexity}

In this section, we provide a deterministic MILP formulation for the problem and address the $\mathcal{N} \mathcal{P}$-hardness of its two (natural) subproblems.

\footnotetext{
2 The works in $[11,18]$ constitute the, to our knowledge, only notable exceptions where VNE is not tackled in a deterministic setting. Differently from the setting we assume in this paper though, both works tackle (heuristically) the online version of VNE where the VN requests arrive over time one by one (or, at most, in batches), whereas, in this work, we assume that the whole set of requests is known beforehand and that admission control is in place. Although our work can clearly be used for the case where a single VN request has to be embedded, we recall that, in this paper, embedding costs are not considered.

3 A preliminary version of this work appeared in [1].
} 


\subsection{Deterministic MILP formulation}

Let us assume, for now, a deterministic setting where all the parameters of the problem are precisely known beforehand. Let the directed graph $G^{0}=\left(V^{0}, A^{0}\right)$ represent the physical network, with node capacities $c_{i}^{0}$ for all $i \in V^{0}$ and link capacities $k_{i j}^{0}$ for all $(i, j) \in A^{0}$. Let $R=1, \ldots,|R|$ be the set of VN requests, with profits $p^{r} \geq 0$ for all $r \in R$. For each $r \in R$, let $V^{r}$ be the set of virtual nodes, each of which endowed with a parameter $\omega_{v}^{r}$ denoting the corresponding node demand and a (possibly sparse) virtual traffic matrix $D^{r} \in \mathbb{R}_{+}^{\left|V^{r}\right| \times\left|V^{r}\right|}$, where each element $d_{v w}^{r} \geq 0$ accounts for the traffic demand between the two virtual nodes $v, w \in V^{r}$. We denote by $V^{0}(r, v) \subseteq V^{0}$ the set of physical nodes to which the virtual node $v \in V^{r}$, pertaining to request $r \in R$, can be mapped due to locality constraints.

The following MILP formulation of VNE is similar to the one proposed in [13], although it neglects some extra aspects (notably, rent-at-bulk) which are outside the scope of this paper. We employ three groups of decision variables: $y_{i j}, x_{v i}^{r}$, and $f_{i j}^{r, v w}$. Let $y^{r} \in\{0,1\}$ take value 1 if the request of index $r \in R$ is accepted and 0 otherwise. Let $x_{v i}^{r} \in\{0,1\}$ be equal to 1 if the virtual node $v \in V^{r}$, pertaining to request $r \in R$, is mapped onto the physical node $i \in V^{0}$, with $y^{r}=0$ otherwise. Let $f_{i j}^{r, v w}$ take value 1 if the traffic between the two virtual nodes $v, w \in V^{r}$, for a request $r \in R$, is routed over the physical link $(i, j) \in A^{0}$, and 0 otherwise. Then, the VNE problem can be cast as the following MILP:

$$
\begin{aligned}
& \max \sum_{r \in R} p^{r} y^{r} \\
& \text { s.t. } \quad \sum_{i \in V^{0}(r, v)} x_{v i}^{r}=y^{r} \\
& \forall r \in R, v \in V^{r} \\
& \sum_{r \in R} \sum_{\substack{v \in V^{r}: \\
i \in V^{0}(r, v)}} \omega_{v}^{r} x_{v i}^{r} \leq c_{i}^{0} \\
& \forall i \in V^{0} \\
& \sum_{r \in R} \sum_{v, w \in V^{r}} d_{v w}^{r} f_{i j}^{v w, r} \leq k_{i j}^{0} \\
& \forall(i, j) \in A^{0} \\
& \sum_{(i, j) \in \delta^{+}(i)} f_{i j}^{v w, r}-\sum_{(j, i) \in \delta^{-}(i)} f_{j i}^{v w, r}=x_{v i}^{r}-x_{w i}^{r} \quad \forall r \in R, v, w \in V^{r}, i \in V^{0} \\
& y^{r} \in\{0,1\} \\
& x_{v i}^{r} \in\{0,1\} \\
& f_{i j}^{v w, r} \in\{0,1\} \\
& \forall r \in R, v \in V^{r}, i \in V^{0}(r, i) \\
& \forall r \in R, v, w \in V^{r},(i, j) \in A^{0} .
\end{aligned}
$$

Constraints (2) enforce that each virtual node is mapped onto a (single) substrate node only if the corresponding request is accepted. Constraints (3) and (4) guarantee that the capacity of each physical node and link is not exceeded. Constraints (5) are flow balance constraints ensuring that the routing of the virtual traffic matrices (which is a function of the mapping of the virtual nodes $v, w \in V^{r}$, as encoded by $x_{v i}^{r}-x_{w i}^{r}$ ) takes place. Each physical node $i \in V^{0}$ acts as a source node if $x_{v i}^{r}=1$ and $x_{w i}^{r}=0$, as a sink node if 
$x_{v i}^{r}=0$ and $x_{w i}^{r}=1$, and as a "regular" intermediate node (i.e., not a source nor a sink node) if $x_{v i}^{r}=x_{w i}^{r}=0$. We remark that, if $x_{v i}^{r}=x_{w i}^{r}=1$, then the two virtual nodes $v, w$ are co-located, i.e., are mapped to the same physical node and, hence, their traffic demands (for both pairs $v, w$ and $w, v$ ) vanish (that is, they do not need to be routed on any physical links). Constraints (6)(8) denote the nature of the variables. Observe that, since $f_{i j}^{v w, r}$ is an integer variable, a single path unsplittable routing is enforced.

We remark that this formulation, as well as the methods that we will introduce in the remainder of the paper, can be directly adapted to the case of splittable routing by just relaxing Constraints (8) into $f_{i j}^{v w, r} \in[0,1]$.

\subsection{VNE and subproblems: computational complexity}

VNE entails the solution of two natural subproblems: node mapping and link mapping. Here, we address the case where both of them allow for admission control. The first subproblem is obtained after dropping Constraints (4) and (5) and the variables reported in (8). The second one is obtained after fixing $x_{v i}^{r}$ for all $r \in R, v \in V^{r}$, and $i \in V^{0}(r, v)$. This way, the first subproblem is a relaxation of $\mathrm{VNE}$, whereas the second one is a restriction.

From a combinatorial point of view, we refer to the first subproblem as a Multi-Knapsack Problem with Grouped items (MKP-G). It is a MultiKnapsack Problem (MKP), i.e., an extension of the classical knapsack problem where more simultaneous knapsacks are present, in which the items are grouped so that, if an item is put into one of the knapsacks, then all the other items in the same group have to be put in some knapsacks as well. From a VNE perspective, each group corresponds to the set of virtual nodes belonging to a virtual network request, the items are virtual nodes, and the knapsacks correspond to physical nodes.

We refer to the second subproblem as to an Unsplittable Multi-Commodity Flow problem with Admission Control (UMCF-AC). In it, the demands of the different commodities can be neglected and a linear function which associates a profit to each accepted flow is maximized. The problem is also subject to "grouping" constraints by which, if the demand for a pair of virtual nodes is routed, then all the demands between pairs belonging to the same group must be routed as well.

In the following, we discuss on the strong $\mathcal{N} \mathcal{P}$-hardness of the two subproblems. The first result is also pointed out in [6]:

Proposition 1 (See [6]) MKP-G is strongly NP-hard.

Proof It suffices to assume that each group contains a single item. Then, MKP$\mathrm{G}$ is equivalent to MKP, whose strong $\mathcal{N} \mathcal{P}$-hardness is shown in [19].

The second result is, to the best of our knowledge, new:

Proposition 2 UMCF-AC is strongly NP-hard. 
Proof Consider the Edge Disjoint Path Problem (EDPP), which calls for $k$ edge disjoint paths in a directed graph between $k$ pairs of nodes. Construct an UMCF-AC instance with the same graph, unit link capacities, unit profits, unit demands, and a group per demand composed of a single element. Then, UMCF-AC admits a solution of value $k$ if and only if the graph admits $k$ edge disjoint paths.

For an sketch of the reduction for a given instance, see Figure 2.

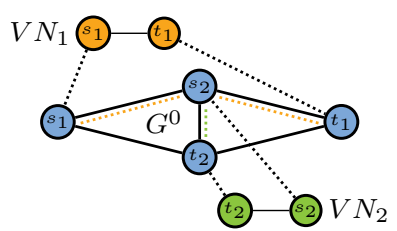

Fig. 2: Reduction from EDPP with $k=2 \mathrm{VNs}$ corresponding to paths being embedded without crossing edges due to the presence of unit link demands and capacities.

Note that, when assuming $\left|V^{0}(r, v)\right|=1$ for all $r \in R$ and $v \in V^{r}$, VNE becomes an instance of UMCF-AC. Therefore, the proposition also implies that VNE is strongly $\mathcal{N} \mathcal{P}$-hard by reduction from EDPP. The two propositions not only illustrate that VNE is a hard problem, but also that both of its naturally occurring subproblems are difficult to solve, at least from a theoretical standpoint.

\section{Addressing the case of data uncertainty}

In many practical applications, it is reasonable to assume that, for each $\mathrm{VN}$, the actual demand of computing resources and traffic may vary, often substantially, over time. For instance, an online gaming or a movie streaming service may have more or less customers, and, therefore, a different resource consumption, depending on its popularity, which clearly changes over time (with, e.g., peaks for new content releases, after an advertising campaign, and so on). This poses a problem from a network reliability point of view, as it can lead to traffic congestion, quality of service degradations, or, even, service disruptions.

Classical approaches to circumvent data uncertainty typically consider a socalled worst case setting, so to guarantee that the network will be operational even for peak values of traffic. Although guaranteeing feasibility, this practice comes at an often unnecessary cost as, in many cases, it is very unlikely for every demand in every VN to simultaneously be at its peak. Indeed, in a number of practical cases, it is reasonable to assume that the probability that all demands simultaneously reach their peak values is fairly small. This is reasonable, in our example, when assuming that new content releases and advertising campaign do not take place for all services at the same time. 
The idea is to look for a solution where the different VNs are provisioned for demands which are smaller than their peak values, thus guaranteeing that the substrate network has sufficient capacity for almost all the traffic configurations, only neglecting a few unlikely cases. This way, we are likely to obtain more profitable solutions where more $\mathrm{VN}$ requests are embedded, thus avoiding costly issues of overprovisioning.

\subsection{A chance-constrained MILP formulation}

One natural way of taking demand uncertainties into account is of interpreting $\omega_{v}^{r}$ and $d_{v w}^{r}$ not as constants, but as (bounded) random variables. In the reminder of this work, we will assume that, for any $r \in R$, each uncertain node demand $\omega_{v}^{r}$ is an independent random variable taking value in the symmetric interval $\left[\bar{\omega}_{v}^{r}-\hat{\omega}_{v}^{r}, \bar{\omega}_{v}^{r}+\hat{\omega}_{v}^{r}\right]$, with nominal (expected) value $\bar{\omega}_{v}^{r}$ and maximum deviation $\hat{\omega}_{v}^{r}$. Similarly, we assume that each uncertain link demand $d_{v w}^{r} \in D^{r}$ takes values in the symmetric interval $\left[\bar{d}_{v w}^{r}-\hat{d}_{v w}^{r}, \bar{d}_{v w}^{r}+\hat{d}_{v w}^{r}\right]$, centered around the nominal (expected) value $\bar{d}_{v w}^{r}$, with a maximum deviation $\hat{d}_{v w}^{r}$.

Let $\epsilon \in[0,1]$ be the probability with which each constraint is required to be satisfied. When requiring the satisfaction of Constraints (3) and (4) with, at least, a probability of $\epsilon$, we obtain the following chance-constrained MILP formulation of VNE:

$$
\begin{aligned}
& \max (1) \\
& \text { s.t. } \operatorname{Pr}\left(\sum_{r \in R} \sum_{v \in V^{r}: i \in V^{0}(r, v)} \omega_{v}^{r} x_{v i}^{r} \leq c_{i}^{0}\right) \geq \epsilon \quad \forall i \in V^{0} \\
& \operatorname{Pr}\left(\sum_{r \in R} \sum_{v, w \in V^{r}} d_{v w}^{r} f_{i j}^{v w, r} \leq k_{i j}^{0}\right) \geq \epsilon \quad \forall(i, j) \in A^{0} \\
& \text { (2), (5), (6), (7), (8). }
\end{aligned}
$$

Note that, if the deterministic Formulation (1)-(8) is solved with worst case data, i.e., by setting each random variable to its maximum value, we obtain an embedding which is also a feasible solution to the chance-constrained Formulation (9)-(12) when solved with any $\epsilon$, and (neglecting zero-measure events) an optimal one for $\epsilon=1$. As we will see with our computational experiments, which we report in Section 5, the objective function value of such solutions is typically very poor.

Clearly, Formulation (1)-(8) can also be solved with nominal data, substituting for each random variable its expected value. Although typically yielding much larger objective function values, this choice corresponds to setting $\epsilon=0$, thus asking for solutions that are feasible only for the zero-measure event where all the uncertain data take a single set of values. As such, these solutions are (theoretically and, often, also in practice) infeasible with probability 1 . 


\subsection{A $\Gamma$-robustness approach}

For most nontrivial probability distributions of the random variables, chanceconstrained problems are, in general, very hard to solve. This is because, to rely on mathematical programming tools, they require a closed-form solution to the integrals corresponding to each probabilistic constraint the derivation of which is, usually, not known. An attractive way to circumvent this drawback, also successfully applied to a number of networking problems, see, e.g., [20], is of recurring to robust optimization and, specifically, to a $\Gamma$-robust approach.

Roughly speaking, the $\Gamma$-robustness model $[21,22]$ assumes that, in any possible realization of the uncertain data (i.e., of the random variables of the chance-constrained model), at most $\Gamma$ coefficients will simultaneously deviate from their nominal value. This model naturally meets the features of VNE if we assume that the number of demands simultaneously reaching their peak values is bounded by $\Gamma$, as $\Gamma$-robust solutions are guaranteed to be feasible for any realization of the uncertain coefficients with at most $\Gamma$ deviations. What is more, $\Gamma$-robustness also establishes, under the sole assumption of independence of the random variables and of symmetry of their intervals, that, if more than $\Gamma$ deviations occur, feasibility will still be retained with a probability corresponding to a monotone increasing function of $\Gamma$. Thus, the model allows to approximate our chance-constrained formulation for an arbitrary $\epsilon$ by selecting a suitable value for $\Gamma$ (see [22] for more details). Most interestingly, the model leads to problems which are computationally much more tractable than those involving chance constraints, as we will show in the following.

In the remainder of the paper, we will refer to the probability that a solution is feasible as protection level (and to its estimation, as observed via computational experiments, as empirical protection level). The adoption of higher values for $\Gamma$, which guarantee a higher protection level, comes at a cost, the so-called price of robustness, as a consequence of the set of feasible solutions becoming smaller for larger values of $\Gamma$.

\subsection{A robust MILP formulation for VNE}

Let us now show how to derive an MILP $\Gamma$-robustness formulation for VNE. We will address the case where, for each node and link, at most $\Gamma$ demands deviate from their nominal value. Equivalently, this corresponds to the case where all but $\Gamma$ demands deviate from their worst case value. We recall that, for $\Gamma=0$, the robust problem corresponds to the original problem with nominal data while, for $\Gamma=\infty$, it corresponds to the original problem with worst case data where all the coefficients simultaneously deviate to their maximum value.

\subsection{1 $\Gamma$-robust MILP formulation for VNE $I$ : node demands}

For convenience, define $\mathcal{V}_{i}^{N}:=\left\{(r, v) \in R \times \cup_{r \in R} V^{r}: i \in V^{0}(r, v)\right\}$. For any physical node $i \in V^{0}$, the set $\mathcal{V}_{i}^{N}$ corresponds to all the request-node pairs $r, v$ 
where the virtual node $v \in V^{r}$ can be mapped to the physical node $i$. Letting $\Gamma \in \mathbb{Z}_{+}$, the (nonlinear) robust counterpart to Constraints (10) is:

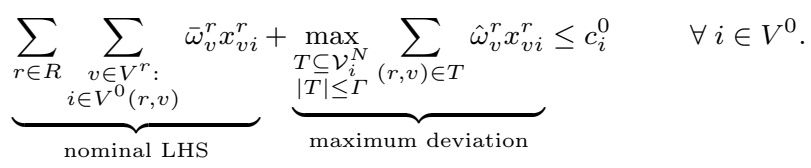

The constraint accounts for the scenario where the $\Gamma$ coefficients with the largest value of $\hat{\omega}_{v}^{r} x_{v i}^{r}$ (those in the set $T$ ) simultaneously deviate. If the constraint is satisfied for the maximum (total) deviation, the nominal constraint will then be satisfied for any realization in the uncertainty set. As originally shown in [22], this nonlinear constraint can be recast in a linear way (i.e., without the need for the internal max operator) with the introduction of a set of auxiliary variables and constraints. We briefly illustrate this in the following.

Let $z^{r v}=1$ if $(r, v) \in T$ and 0 otherwise. Assuming that $x_{v i}^{r}$ is fixed, the inner maximization problem can be cast as the following Linear Program (LP), whose dual variables are reported in brackets:

$$
\begin{array}{lll}
\max & \sum_{r \in R} \sum_{\substack{v \in V^{r}: \\
i \in V^{0}(r, v)}}\left(\hat{\omega}_{v}^{r} x_{v i}^{r}\right) z_{i}^{r v} & \\
\text { s.t. } & \sum_{r \in R} \sum_{\substack{v \in V^{r}: \\
i \in V^{0}(r, v)}} z_{i}^{r v} \leq \Gamma & {\left[\pi_{i}\right]} \\
& z_{i}^{r v} \in[0,1] \quad \forall r \in R, v \in V^{r}: i \in V^{0}(r, v) & {\left[\rho_{i}^{r v}\right] .}
\end{array}
$$

Note that, since the problem has a totally unimodular constraint matrix, $z_{i}^{r v}$ will be integer in any optimal solution. The LP dual reads:

$$
\begin{array}{lll}
\min & \Gamma \pi_{i}+\sum_{r \in R} \sum_{\substack{v \in V^{r}: \\
i \in V^{0}(r, v)}} \rho_{i}^{r v} & \\
& & \\
\text { s.t. } & \pi_{i}+\rho_{i}^{r v} \geq \hat{\omega}_{v}^{r} x_{v i}^{r} & \forall r \in R, v \in V^{r}: i \in V^{0}(r, v) . \\
& \pi_{i} \geq 0, \quad \rho_{i}^{r v} \geq 0 & \forall r \in R, v \in V^{r}: i \in V^{0}(r, v) .
\end{array}
$$

By LP duality, any feasible solution to (16)-(18) has an objective function value at least as large as that of an optimal solution to (13)-(15). Therefore, for each $i \in V^{0}$, the robust counterpart to Constraint (10) corresponds to:

$$
\sum_{r \in R} \sum_{v \in V^{r}: i \in V^{0}(r, v)}\left(\bar{\omega}_{v}^{r} x_{v i}^{r}+\rho_{i}^{r v}\right)+\Gamma \pi_{i} \leq c_{i}^{0}
$$

together with Constraints (17) and the variables in (18). 
3.3.2 $\Gamma$-robust MILP formulation for VNE II: traffic (link) demands

Let $\mathcal{V}^{L}:=\left\{(r, v, w): r \in R, v, w \in V^{r}\right\}$. Letting $\Gamma \in \mathbb{Z}_{+}$, the (nonlinear) robust counterpart to Constraint (11), for all $(i, j) \in A^{0}$, reads:

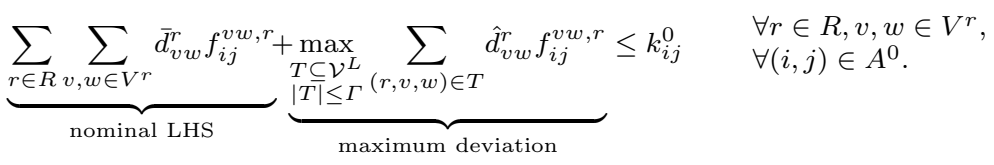

Similarly to the node case, a linear reformulation can be obtained after introducing the variables $\pi_{i j}, \rho_{i j}^{v w, r} \geq 0$ for all $r \in R, v, w \in V^{r}$ and, for all $(i, j) \in A^{0}$, the constraints:

$$
\begin{array}{ll}
\sum_{r \in R} \sum_{v, w \in V^{r}}\left(\bar{d}_{v w}^{r} f_{i j}^{v w, r}+\rho_{i j}^{v w, r}\right)+\Gamma \pi_{i j} \leq k_{i j}^{0} & \\
\pi_{i j}+\rho_{i j}^{v w, r} \geq \hat{d}_{v w}^{r} f_{i j}^{v w, r} & \forall r \in R, v, w \in V^{r} \\
\pi_{i j} \geq 0, \quad \rho_{i j}^{v w, r} \geq 0 & \forall r \in R, v, w \in V^{r} .
\end{array}
$$

The complete $\Gamma$-robust formulation for VNE is obtained from that in (1)-(8) after substituting for Constraints (3) and (4) their robust counterparts.

\section{Heuristics for the $\boldsymbol{\Gamma}$-robust VNE problem}

Although much more tractable than its original chance-constrained counterpart (as it "only" requires the solution of an MILP), the $\Gamma$-robust version of VNE is still, as we will see in Section 5, very hard to solve for large instances within a reasonable computing time. Hence, in this section we propose two heuristics approaches to produce good-quality robust solutions at a smaller computational effort. Both approaches rely on splitting the VNE problem into the robust counterparts to the two subproblems which we mentioned before, which are then solved sequentially within a given time limit.

\subsection{Two-phase heuristic}

First, let us outline our two-phase method. In the first phase, we carry out admission control and $\Gamma$-robust node embedding, but neglect link mapping and link capacities. In the second phase, we complete the partial solution found in phase one by looking for a $\Gamma$-robust link mapping for the accepted VNs (assuming that their node mapping is fixed, as found in the first phase), while still allowing for VN rejections. Similar ideas (node mapping in the first phase, routing in the second one) have already been applied to VNE, see for instance [8], but, differently from other methods, in our case we allow for VN rejections in both phases, include the robustness aspect in each phase, and solve each subproblem as an MILP. In this sense, due to entailing the solution 
of two MILPs at each iteration, our algorithm could be classified as a math heuristic.

\subsubsection{Phase one subproblem}

In the first phase, we restrict VNE to the $\Gamma$-robust node embedding subproblem with admission control. Formally, this amounts to the $\Gamma$-robust MILP:

$$
\begin{aligned}
& \max \sum_{r \in R} p^{r} y^{r} \\
& \text { s.t. } \sum_{i \in V^{0}(r, v)} x_{v i}^{r}=y^{r} \quad \forall r \in R, v \in V^{r} \\
& \sum_{r \in R} \sum_{v \in V^{r}: i \in V^{0}(r, v)}\left(\bar{\omega}_{v}^{r} x_{v i}^{r}+\rho_{i}^{r v}\right)+\Gamma \pi_{i} \leq c_{i}^{0} \quad \forall i \in V^{0} \\
& \pi_{i}+\rho_{i}^{r v} \geq \hat{\omega}_{v}^{r} x_{v i}^{r} \quad \forall r \in R, v \in V^{r}, i \in V^{0}(r, v) \\
& y^{r} \in\{0,1\} \\
& x_{v i}^{r} \in\{0,1\} \\
& \forall r \in R \\
& \pi_{i} \geq 0, \rho_{i}^{r v} \geq 0 \\
& \forall r \in R, v \in V^{r}, i \in V^{0}(r, v) \\
& \forall r \in R, v \in V^{r}, i \in V^{0}(r, v) .
\end{aligned}
$$

This subproblem is the $\Gamma$-robust counterpart to the MKP-G problem that we introduced in Section 2. While, as we have shown, MKP-G is strongly $\mathcal{N} \mathcal{P}$ hard, it is fairly easier to solve than the whole $\Gamma$-robust VNE problem, as we will see in Section 5. We remark that, by construction, optimal solutions to this subproblem provide upper bounds to the $\Gamma$-robust version of VNE.

\subsubsection{Phase two subproblem}

Let $(\tilde{y}, \tilde{x})$ be a solution to the first phase problem and let $\tilde{R}:=\left\{r \in R: \tilde{y}^{r} \neq 0\right\}$ be the subset of requests that have been accepted in the first phase. Also define $\tilde{\Delta}^{r} \in\{0,1\}^{\left|V^{r}\right| \times\left|V^{r}\right|}$ so that $\tilde{\delta}_{v w}^{r}=0$ if and only if $\tilde{x}_{v i}^{r}=\tilde{x}_{w i}^{r}=1$ for some $i \in V^{0}$ (recall that the corresponding flow vanishes due to co-location). Then, the second phase problem corresponds to the following $\Gamma$-robust MILP:

$$
\begin{aligned}
& \max \sum_{r \in R^{\prime}} p^{r} y^{r} \\
& \text { s.t. } \sum_{(i, j) \in \delta^{+}(i)} f_{i j}^{v w, r}-\sum_{(j, i) \in \delta^{-}(i)} f_{j i}^{v w, r}= \begin{cases}y^{r} \text { if } \tilde{x}_{v i}^{r}=1 \\
-y^{r} \text { if } \tilde{x}_{w i}^{r}=1 \\
0 \quad \text { else } & \forall i \in V^{0}, r \in R^{\prime}, \\
0 v, w \in V^{r}: \tilde{\delta}_{v w}^{r}=1\end{cases} \\
& \sum_{r \in R^{\prime}} \sum_{\substack{v, w \in V^{r}: \\
\tilde{\delta}^{r}=1}}\left(\bar{d}_{v w}^{r} f_{i j}^{v w, r}+\rho_{i j}^{v w, r}\right)+\Gamma \pi_{i j} \leq k_{i j}^{0} \quad \forall(i, j) \in A^{0} \\
& \pi_{i j}+\rho_{i j}^{v w, r} \geq \hat{d}_{v w}^{r} f_{i j}^{v w, r} \quad \forall r \in R^{\prime}, v, w \in V^{r}: \tilde{\delta}_{v w}^{r}=1,(i, j) \in A^{0} \\
& y^{r} \in\{0,1\} \\
& f_{i j}^{v w, r} \in\{0,1\} \\
& \forall r \in R^{\prime} \\
& \pi_{i j} \geq 0, \rho_{i j}^{v w, r} \geq 0 \\
& \forall r \in R^{\prime}, v, w \in V^{r}: \tilde{\delta}_{v w}^{r}=1,(i, j) \in A^{0} \\
& \forall r \in R^{\prime}, v, w \in V^{r}: \tilde{\delta}_{v w}^{r}=1,(i, j) \in A^{0} .
\end{aligned}
$$


The subproblem is the $\Gamma$-robust counterpart to the UMCF-AC problem that we introduced in Section 2. In spite of its strong $\mathcal{N} \mathcal{P}$-hardness, as we will see in Section 5, this subproblem will be much easier to solve, for all instances that we will consider, than MKP-G.

We note that, by solving the phase one and phase two subproblems in sequence with the same value of $\Gamma$, the heuristic that we introduced always provides a lower bound (i.e., a feasible solution) to the $\Gamma$-robust VNE problem.

\subsubsection{Revised phase one subproblem}

Preliminary experiments have shown that, in many cases, more than $50 \%$ of the requests accepted in the first phase are then discarded in the second phase. This is a consequence of the fact that the phase one subproblem is oblivious of the routing aspect. Among its feasible solutions, we would indeed prefer one where pairs of virtual nodes sharing a traffic demand are mapped to physical nodes which are as close as possible. This is because, the more links are used in the routing, the higher the consumption of link capacity in the substrate network will be. A sketch of this simple observation can be found in Figure 3.

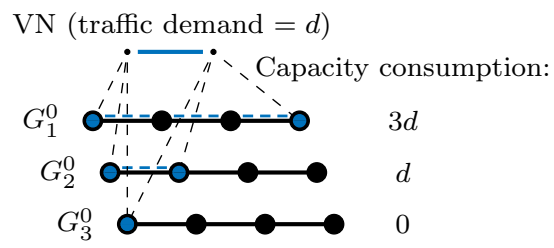

Fig. 3: Three embeddings of a single VN requiring a different amount of physical link capacity. Note how, in case of co-location, the flow between source and sink nodes vanishes.

To circumvent this drawback, we restrict the feasible region of the first phase subproblem to solutions where pairs of virtual nodes sharing a traffic demand are mapped to physical nodes that are not too far away from each other, thus, hopefully, reducing the number of rejections in phase two. For the purpose, we cluster the virtual node pairs $v, w \in V^{r}$ of any $\mathrm{VN}$ request of index $r \in R$ into a set $\mathcal{C}$ of categories, depending on their traffic demand values $d_{v w}^{r}$. For each category $C \in \mathcal{C}$, we introduce a parameter $z_{C}$ which describes the maximum distance, in terms of number of links, that we allow between the physical nodes onto which $v$ and $w$ can be mapped.

More formally, for any two physical nodes $i, j \in V^{0}$, let $\sigma(i, j)$ be the length of a shortest path (in terms of number of links) in $G^{0}$ between them. We partition the different pairs of virtual nodes into three categories, based on the magnitude of their traffic demands: $L$ (for low), $M$ (for medium), and $H$ (for 
high). Given the three corresponding parameters $z_{L}, z_{M}, z_{H} \in \mathbb{Z}_{+}$, we introduce the following distance-bounding constraints to the phase one problem:

$$
x_{v i}^{r}+x_{w j}^{r} \leq 1 \quad \forall r \in R, v, w \in V^{r}, i, j \in V^{0}:\left\{\begin{array}{l}
\sigma(i, j)>z_{L} \wedge\{v, w\} \in L \\
\sigma(i, j)>z_{M} \wedge\{v, w\} \in M \\
\sigma(i, j)>z_{H} \wedge\{v, w\} \in H .
\end{array}\right.
$$

The results obtained with this heuristic will be discussed in Section 5 .

\subsection{Adaptive heuristic}

While the two-phase heuristic very effectively provides good quality solutions in a short amount of computing time (see Section 5), its success heavily depends on a good choice of its input parameters $z_{L}, z_{M}, z_{H}$. To avoid the need for finding a suitable choice of such parameters a priori, we now propose an adaptive algorithm in which a suitable parameter setting is chosen automatically.

The method solves a sequence of $\Gamma$-robust phase one and phase two subproblems. If, at any iteration, the phase two subproblem terminates accepting all the requests that were accepted in phase one, it halts. If not, it looks for a pair of virtual nodes which, if their VN were accepted, would consume the largest quantity of link capacity, adds a constraint similar to Constraint (38) to the phase one subproblem to reduce the corresponding amount of physical link capacity consumption, and iterates until a time or iteration limit is met.

For any virtual node $v$, denote by $i(v)$ the physical node to which $v$ has been mapped in the last iteration. At each iteration and for each request $r \in R$, we associate to each pair of virtual nodes $\{v, w\}$, with $v, w \in V^{r}$, a value equal to the product between their traffic demand and the distance $\sigma(i(v), i(w))$ between the corresponding physical nodes $i(v), i(w)$ in terms of number of links. Then, for each request of index $r \in R$, we identify the following virtual node pair:

$$
\left(v^{\prime}, w^{\prime}\right):=\underset{v, w \in V^{r}}{\operatorname{argmax}}\left\{\max \left\{d_{v w}^{r}, d_{w v}^{r}\right\} \cdot \sigma(i(v), i(w))\right\} .
$$

Note that, due to employing the shortest path measure in term of number of links, the expression which is maximized corresponds to the minimum physical resource consumption that would correspond to any embedded virtual pair.

Then, to impose a mapping to a closer pair of physical nodes, we add to the first phase problem, for the current triple $\left(r, v^{\prime}, w^{\prime}\right)$, the constraints:

$$
x_{v^{\prime} i}^{r}+x_{w^{\prime} j}^{r} \leq 1 \quad \forall i, j \in V^{0}: \sigma(i, j)>\left\lceil\frac{\sigma\left(i\left(v^{\prime}\right), i\left(w^{\prime}\right)\right)}{2}\right\rceil
$$

if $\sigma\left(i\left(v^{\prime}\right), i\left(w^{\prime}\right)\right)>4$, and the constraints:

$$
x_{v^{\prime} i}^{r}+x_{w^{\prime} j}^{r} \leq 1 \quad \forall i, j \in V^{0}: \sigma(i, j)>\sigma\left(i\left(v^{\prime}\right), i\left(w^{\prime}\right)\right)-1
$$

if $\sigma\left(i\left(v^{\prime}\right), i\left(w^{\prime}\right)\right) \leq 4$.

The pseudocode for the adaptive heuristic is reported in Algorithm 1. 


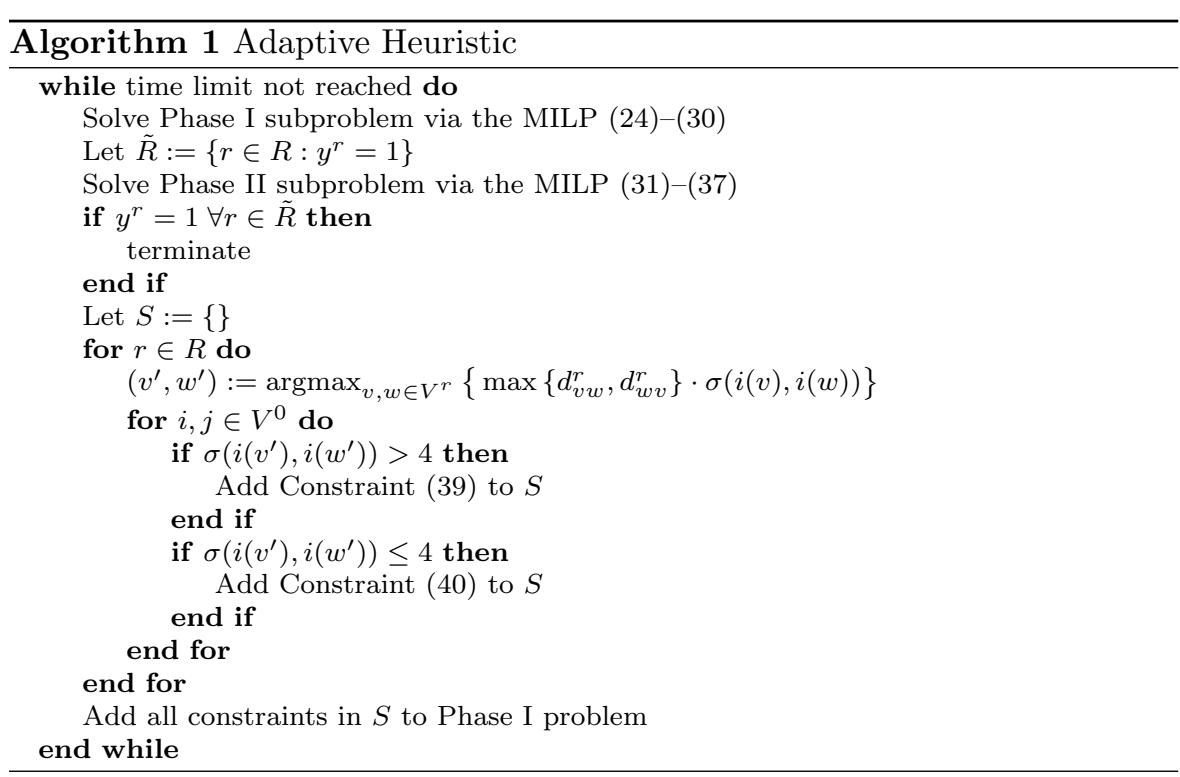

\section{Computational results}

Our computations are carried out on an Intel(R) Core(TM) i7-3770 CPU @ $3.40 \mathrm{GHz}$ with $32 \mathrm{~GB}$ RAM. We employ the state-of-the-art MILP solver CPLEX 12.4, relying on AMPL as modeling language. We set a time limit of 3600 seconds for the exact $\Gamma$-robust MILP formulations, adopting a much shorter time limit of 300 seconds per subproblem in both the two-phase and the adaptive heuristics. The latter is run for, at most, 12 iterations.

As physical networks, we consider four instances of similar size and density, all taken from the SNDlib [23] and transformed into directed graphs with antiparallel links: Abilene (12 nodes, 30 links), Atlanta $(15,44)$, NobelUs $(14,42)$, and PolskA $(12,36)$. The physical node capacities are randomly drawn from the tuple $(10,50,100,500)$, with a probability of $(0.1,0.4,0.4,0.1)$. Physical link capacities are set to 500 for all the edges.

We consider VN requests with 12 virtual nodes, a profit chosen uniformly at random between 20 and 100, and a random topology with a link density of 0.5 . As to the locality aspect, we construct each set $V^{0}(r, v)$ by first sampling uniformly at random a cardinality factor $\alpha_{v}^{r}$ from the interval $\left[\frac{1}{2}, 1\right]$ and then adding node $i \in V^{0}$ to $V^{0}(r, v)$ with a probability $\alpha_{v}^{r}$.

For the virtual node and traffic demands, we mimic a case where historical data are available, creating a historical sequence of 100 data sets. First, for each uncertain coefficient $\omega_{v}^{r}$ or $d_{v w}^{r}$, we sample a value from the tuple $(10,50,100,500)$ (scaled by 0.04 for nodes and 0.06 for links) uniformly at random, with a probability of $(0.1,0.4,0.4,0.1)$. The historical sequence for that coefficient is then constructed by adding to the previously sampled value a Gaussian error with zero mean and a standard deviation equal to three 
times the original value, for each of the 100 snapshots in the sequence, forcing any demand value thus obtained to 0 if negative. Finally, the nominal node and traffic demands $\bar{w}_{v}^{r}$ and $\bar{d}_{v w}^{r}$ are computed as the arithmetic average over the 100 snapshots, computing $\hat{\omega}_{v}^{r}$ and $\hat{d}_{v w}^{r}$ as the largest deviations w.r.t. $\bar{w}_{v}^{r}$ and $\bar{d}_{v w}^{r}$ over the historical sequence.

We generate the instances with an increasing number of requests, that is $|R| \in\{5,6,7,8,9,10,12,14,16,18,20,24,28,32\}$. They are constructed incrementally for each topology, so that every instance of a given topology with $r$ requests contains the same requests as an instance with the same topology and $r^{\prime}<r$ requests, plus $r-r^{\prime}$ additional ones. As a consequence, the value of an optimal solution for any given topology is a nondecreasing function of $|R|$. During the generation of the instances, we choose the random seeds so that the sequence of $\mathrm{VN}$ requests is different for each physical topology. The data set thus constructed is composed of 56 instances.

In the following, we will compare the solutions obtained via the different methods w.r.t. their objective function value and their empirical protection level. The latter is defined as the number of snapshots, in the historical sequence of each instance, for which no node or link capacity constraint is violated by the robust solution that we have found.

\subsection{Exact nominal and worst case solutions}

To better motivate the relevance of data uncertainty for VNE, as well as the profitability of a robust optimization approach, we first evaluate the solutions obtained for worst case demands (i.e., for $\Gamma=\infty$ and $\epsilon=1$ ) and average demands (for $\Gamma=0$ and $\epsilon=0$ ), as mentioned in Section 3 .

Complete results for the full data set are reported in Table 1. The table shows that the instances with the worst case data are quite harder to solve than those with average data (with, overall, an average gap of $32 \%$ versus one of $0 \%$, and an average computing time 3.5 times larger), thus showing that the problem gets more difficult for a higher load. More precisely, out of 56 instances, while only 8 instances cannot be solved to optimality with average data (with an average gap of $2.75 \%$ ), this number increases to 33 instances with worst case data (with an average gap of $53 \%$ ).

As we anticipated in Section 3, although the objective function values with average data are much larger than those for the worst case, for the majority of instances the corresponding solutions are infeasible in almost all the snapshots of the historical sequence. On the contrary, the solutions with worst case data are always feasible, but at the cost of a very poor objective function value. This is illustrated, for the ABILENE instances, in Figure 4.

\subsection{Exact solutions via the $\Gamma$-robust MILP formulation}

We now illustrate the results obtained with the $\Gamma$-robust MILP formulation, first focusing on the ABILENE instances. We assume $\Gamma \in\{1,2,3\}$, adopting the 
Table 1: Results for the deterministic MILP formulation obtained within 3600 seconds with nominal (average) and worst case data. Entries are rounded to the nearest integer.

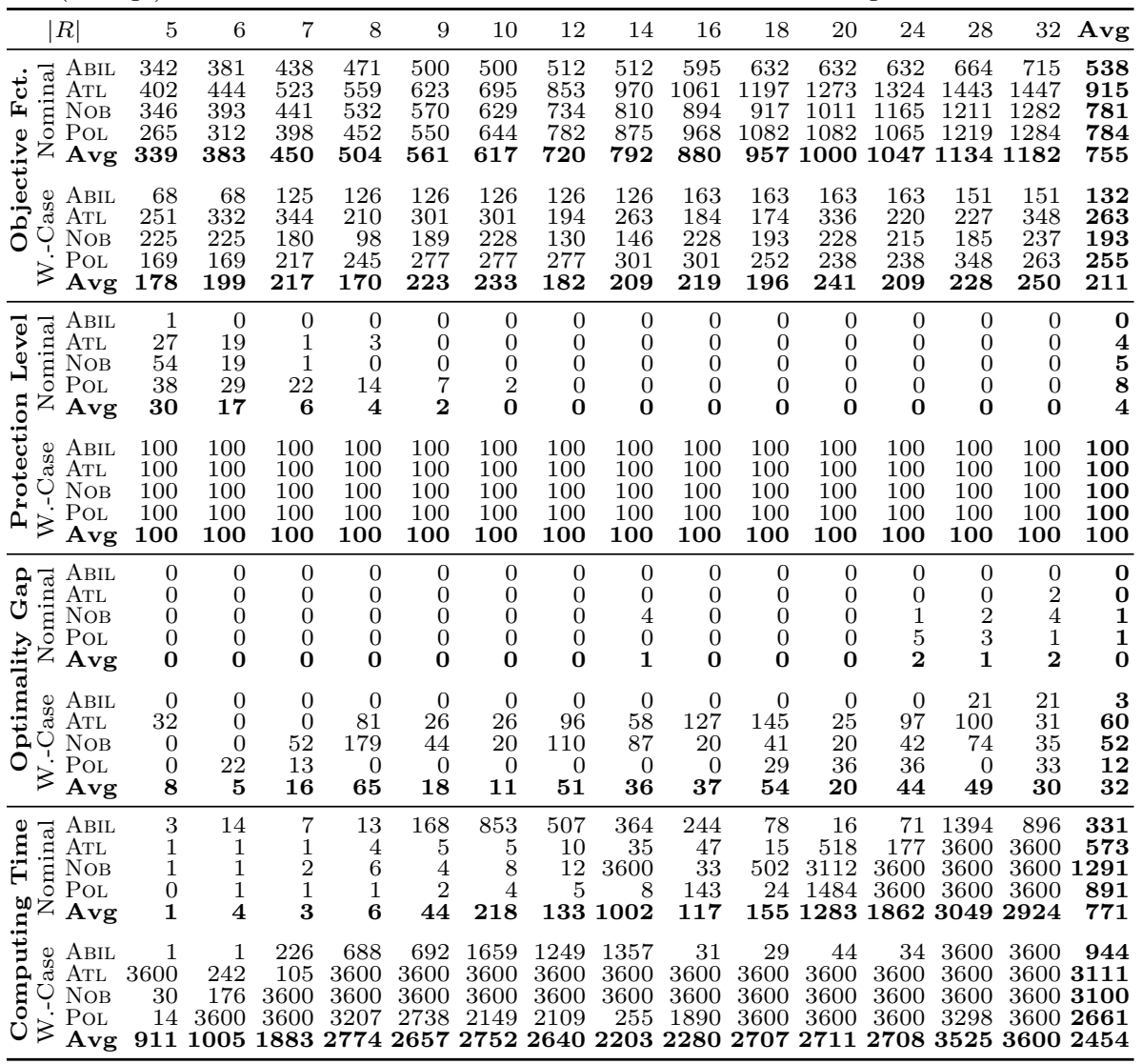



(a)

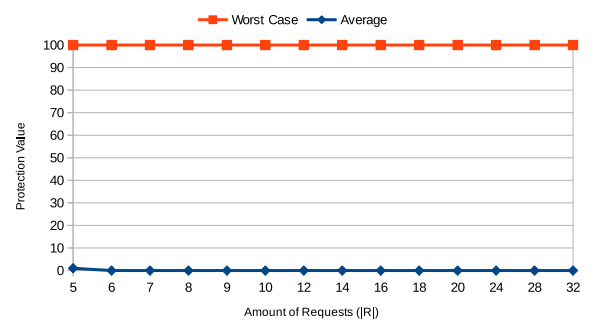

(b)

Fig. 4: (a) Objective function values and (b) protection values for the worst case and average case, reported as a function of $|R|$, for the ABILENE instances. 
same value for both the node and link capacity constraints. We do not report further results for larger values of $\Gamma$ as, in our experiments, we achieve a very high empirical protection level already for $\Gamma=3$.

Figure 5 (a) reports the objective function value for different values of $\Gamma$, as a function of $|R|$. Note that the value of an optimal $\Gamma$-robust solution should be between the average and worst case ones and that such value should be larger for smaller values of $\Gamma$. As we can see, this is not always the case. E.g., we observe that, for $|R|=16$ and $\Gamma=3$, as a consequence of prematurely reaching the time limit, we achieve a strictly smaller objective function value than for the worst case. In general, it seems that, with many requests $(|R|>14)$ and for increasing values of $\Gamma$, the $\Gamma$-robust formulations are more and more difficult to solve. As an example, observe that, for $|R|=28$, no solution is found for $\Gamma \in\{1,2,3\}$, while the solution for $|R|=32$ and $\Gamma=1$ has a smaller value than that for the same $\Gamma$ and $|R|=24$. This is better shown in Figure 5 (b), which reports the optimality gap of the solutions (truncated to 500 for illustration purposes), showing that, for instances with a large $|R|$ and for larger values of $\Gamma$, the exact approach based on the $\Gamma$-robust formulation does not scale well.

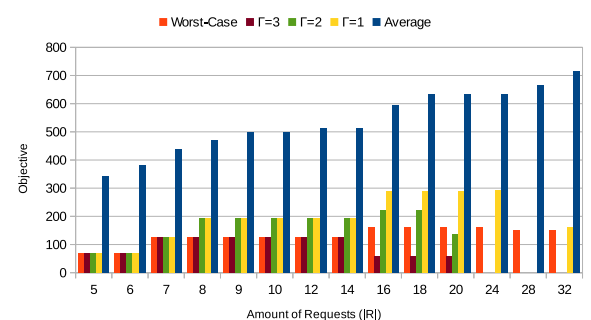

(a)

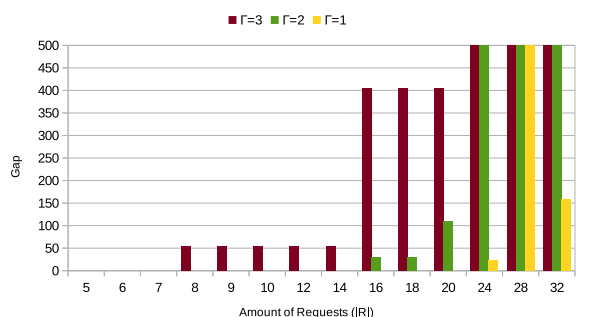

(b)

Fig. 5: (a) Objective function values for the ABILENE instances, obtained with the different models and reported as a function of $|R|$. (b) Gaps for the ABILENE instances.

We obtain qualitatively comparable results also for the other topologies, as reported in Table 2 . Indeed, when considering the full data set (56 instances) with three values of $\Gamma$ (168 VNE problems in total), in 119 cases we cannot find an optimal solution within the time limit, registering an average gap of $76 \%$ (only considering the instances where the gap is finite). In 101 cases, not even a nontrivial solution (i.e., one where at least a single VN is accepted) is found (thus, the gap is infinite). This shows that, when compared to the average and worst case data, with $\Gamma$-robustness we obtain much harder problems.

\subsection{Two-phase and adaptive heuristics}

Let us now consider the two heuristic methods. Differently from the exact case, where we consider $\Gamma \in\{0,1,2,3\}$ for both node and link capacity constraints, 
Table 2: Results for the exact MILP $\Gamma$-robust formulation obtained within 3600 seconds. Entries are rounded to the nearest integer.

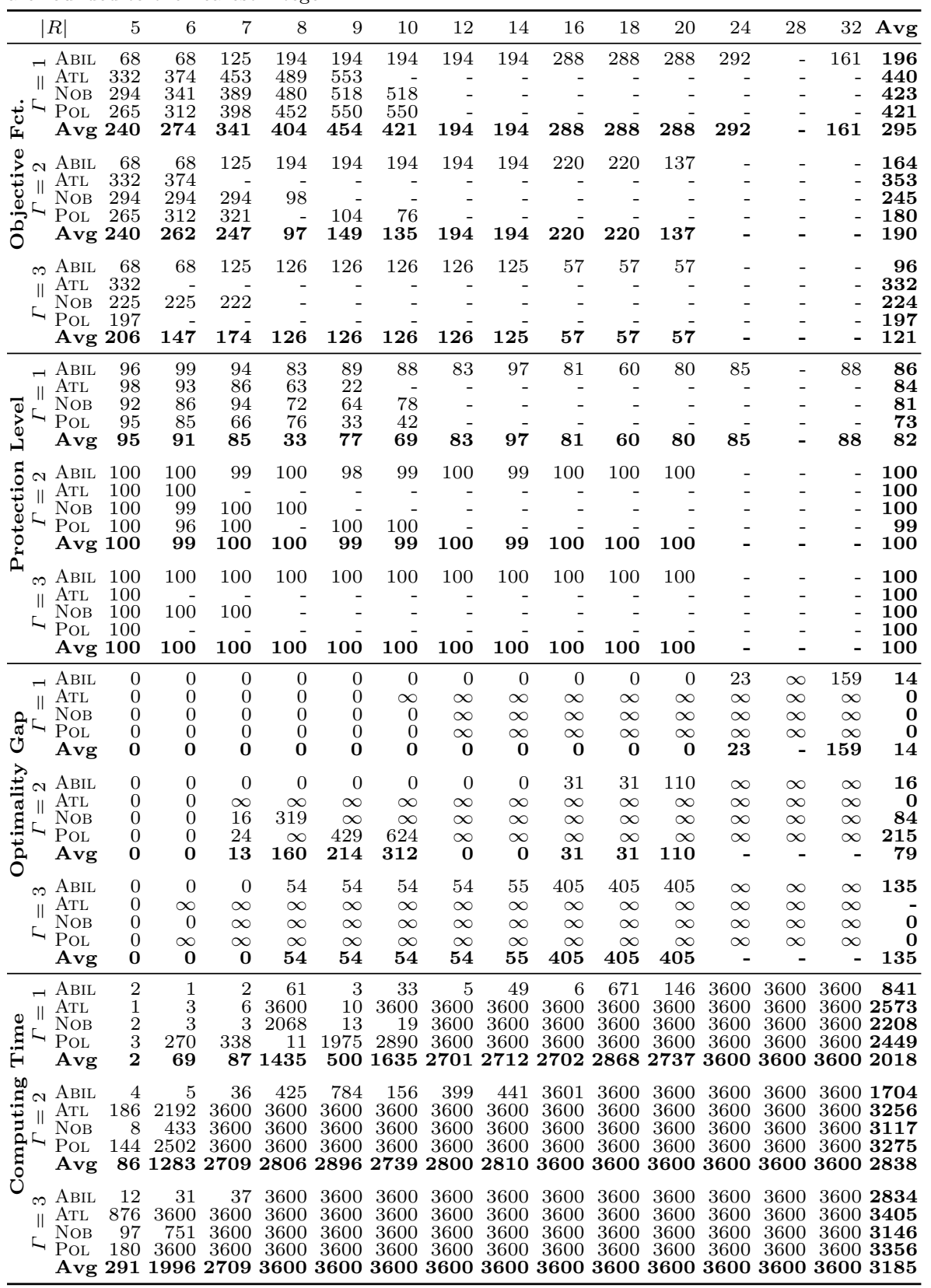


in the two heuristics methods we always set $\Gamma=0$ for the second subproblem (which carries out the link mapping). This is because, even without explicitly accounting for robustness in it, we still obtain solutions with a very high empirical protection level, as we will better illustrate in the following.

Let us first focus on the two-phase heuristic. As described in Section 4, we cluster the virtual nodes pairs into the three categories $L, M, H$ (low, medium, and high). Each pair $v, w \in V^{r}$ belongs to $H$ if $d_{v w}^{r} \geq 50$, to $M$ if $10 \leq d_{v w}^{r}<50$, and to $L$ otherwise. To assess the sensitivity of the w.r.t. the parameters $z_{L}, z_{M}$, and $z_{H}$, we illustrate the results obtained with the method for all the combinations of $z_{L}, z_{M}, z_{H} \in\left\{\left|V^{0}\right|, \frac{\left|V^{0}\right|}{2}, \frac{\left|V^{0}\right|}{4}, 2,1\right\}$, with $z_{L} \leq z_{M} \leq z_{H}$. Table 3 reports a comparison over all parameter settings, aggregated over all substrate networks and all $\Gamma$ values. Let $S O L(\Gamma, s, r, p)$ be the solution value of the two-phase heuristic for a given $\Gamma \in G:=\{0,1,2,3\}$, substrate network $s \in S:=\{$ abilene, atlanta, nobel-us, polska\}, number of requests $r=|R| \in\{5,6,7,8,9,10,12,14,16,18,20,24,28,32\}$, and parameter setting $p \in P$. Then, for a fixed $r$ and parameter setting $p_{0}$, each entry of Table 3 is computed as $\frac{1}{|G||S||P|} \sum_{\Gamma \in G} \sum_{s \in S} \sum_{p \in P} \frac{S O L(\Gamma, s, r, p)}{\operatorname{SOL}\left(\Gamma, s, r, p_{0}\right)}$. Thus, each entry describes the relative quality of the solutions obtained with parameter setting $p_{0}$, compared to the solutions obtained with all other parameter settings. The lower the value, the better $p_{0}$ performs w.r.t. the others (the results for the best setting, on average, over all the instances are underlined). As we can see, the unique "winner" is $z_{L}=\left|V^{0}\right|, z_{M}=2$, and $z_{H}=1$.

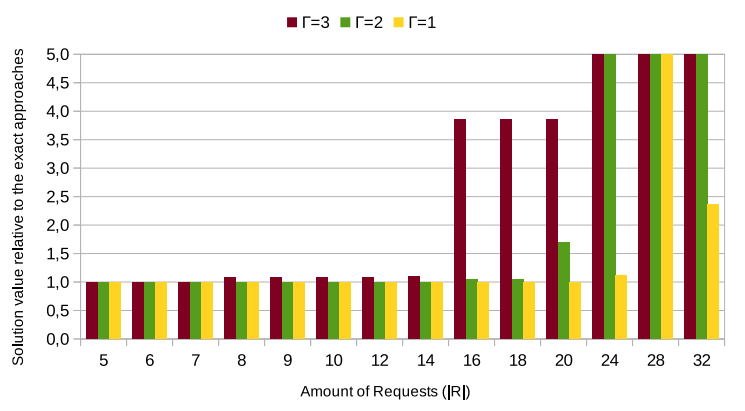

Fig. 6: Objective function value ratio between the best solution found via the two-phase heuristic $\left(z_{L}=\left|V^{0}\right|, z_{M}=2\right.$, and $\left.z_{H}=1\right)$ and the exact formulation, reported as a function of $|R|$, for the ABILENE instances.

We now compare the results for the "winning" parameter setting to the exact approach. We first illustrate the results for the ABILENE instances. In Figure 6 , we report the ratio between the solution values found heuristically and the best ones obtained via the exact formulation (in case no nonzero solution is found with the exact method, we set this ratio to 5.0). Interestingly, for $\Gamma=1$, the heuristic provides competing solutions to those obtained via the exact approach for all the ABILENE instances. For the first half of the instances (those with $|R| \leq 14$ ), the heuristic achieves a comparable objective 
Table 3: Performance ratio of each parameter setting $z_{L}, z_{M}, z_{H} \in\left\{\left|V^{0}\right|, \frac{\left|V^{0}\right|}{2}, \frac{\left|V^{0}\right|}{4}, 2,1\right\}$ with $z_{L} \leq z_{M} \leq z_{H}$, for each $|R|$. The results for the, on average, best setting over all the instances are underlined.

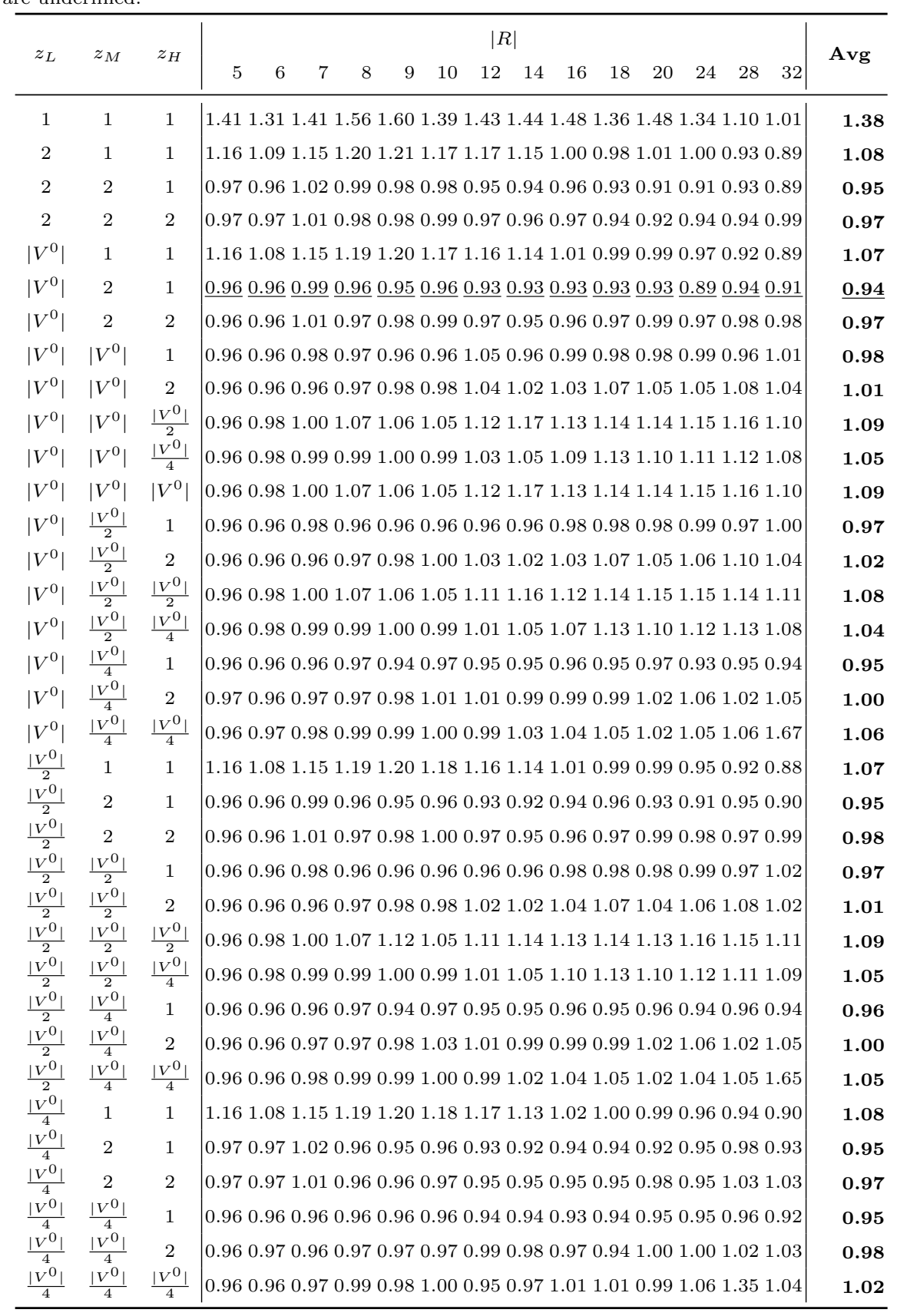


Table 4: Detailed results for the two-phase heuristic with $z_{L}=\left|V^{0}\right|, z_{M}=2$, and $z_{H}=1$.

\begin{tabular}{|c|c|c|c|c|c|c|c|c|c|c|c|c|c|c|c|c|c|}
\hline & & $|R|$ & 5 & 6 & 7 & 8 & 9 & 10 & 12 & 14 & 16 & 18 & 20 & 24 & 28 & 32 & Avg \\
\hline \multirow{20}{*}{ 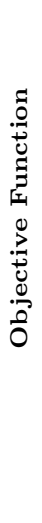 } & 0 & $\mathrm{ABI}$ & 342 & 381 & 438 & 471 & 500 & 500 & 512 & 512 & 595 & 632 & 632 & 632 & 664 & 715 & 538 \\
\hline & 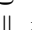 & ATL & 402 & 444 & 523 & 559 & 623 & 695 & 853 & 900 & 988 & 1001 & 1119 & 1091 & 1179 & 1090 & 9 \\
\hline & is & NoB & 346 & 393 & 441 & 532 & 570 & 629 & 696 & 771 & 782 & 841 & 851 & 997 & 1105 & 1128 & 720 \\
\hline & -1 & POL & 265 & 312 & 398 & 452 & 522 & 644 & 702 & 718 & 726 & 714 & 795 & 873 & 774 & 866 & 626 \\
\hline & & Avg & 339 & 383 & 450 & 504 & 554 & 617 & 691 & 725 & 773 & 797 & 849 & 898 & 931 & 950 & 676 \\
\hline & -1 & $\mathrm{ABI}$ & 68 & 68 & 125 & 194 & 194 & 194 & 194 & 194 & 288 & 288 & 288 & 360 & 360 & 381 & 228 \\
\hline & 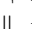 & ATL & 332 & 374 & 453 & 489 & 553 & 625 & 783 & 900 & 912 & 960 & 949 & 925 & 892 & 903 & 718 \\
\hline & $\pi$ & NoB & 294 & 341 & 389 & 480 & 518 & & 555 & 631 & 621 & 636 & 606 & 724 & 650 & 637 & 543 \\
\hline & -1 & POL & 265 & 312 & 398 & 452 & 522 & 550 & 570 & 606 & 642 & 633 & 602 & 573 & 651 & 732 & 536 \\
\hline & & Avg & 240 & 274 & 341 & 404 & 447 & 472 & 526 & 583 & 616 & 629 & 611 & 646 & 638 & 663 & 506 \\
\hline & v & $\mathrm{ABI}$ & 68 & 68 & 125 & 194 & 194 & 194 & 194 & 194 & 220 & 220 & 220 & 256 & 277 & 277 & 193 \\
\hline & $\|$ & ATL & 332 & 374 & 453 & 489 & 511 & & 605 & 655 & 686 & 697 & 703 & 683 & 737 & 730 & 10 \\
\hline & & NoB & 294 & 294 & 342 & 366 & 402 & 402 & 402 & 395 & 401 & 360 & 404 & 467 & 411 & 410 & 382 \\
\hline & & $\mathrm{POL}$ & 265 & 312 & 368 & 394 & 454 & 406 & 478 & 500 & 491 & 526 & 554 & 573 & 601 & 606 & 466 \\
\hline & & Avg & 240 & 262 & 322 & 361 & 390 & 380 & 420 & 436 & 450 & 451 & 470 & 495 & 507 & 506 & 406 \\
\hline & $m$ & ABI & 68 & 68 & 68 & 126 & 126 & 126 & 126 & 126 & 220 & 220 & 220 & 220 & 220 & 220 & 154 \\
\hline & 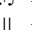 & ATL & 332 & 332 & 411 & 447 & 447 & 447 & 459 & 497 & 535 & 560 & 497 & 585 & 604 & 582 & 481 \\
\hline & & NoB & 225 & 225 & 273 & 282 & 320 & 320 & 320 & 319 & 320 & 320 & 330 & 457 & 366 & 354 & 317 \\
\hline & -1 & POL & 197 & 244 & 322 & 346 & 397 & 406 & 456 & 500 & 500 & 526 & 526 & 475 & 500 & 585 & 427 \\
\hline & & Avg & 206 & 217 & 269 & 300 & 323 & 325 & 340 & 361 & 394 & 407 & 393 & 434 & 423 & 435 & 345 \\
\hline \multirow{20}{*}{ 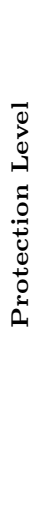 } & 0 & $\mathrm{ABI}$ & 0 & 1 & 1 & 0 & 1 & 2 & 0 & 0 & 0 & 0 & & & 0 & 0 & 1 \\
\hline & 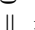 & ATL & 3 & 11 & 6 & 8 & 1 & 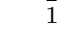 & 2 & & & 0 & & & & 0 & 2 \\
\hline & & NoB & 37 & 39 & 28 & 9 & 6 & 3 & 1 & & 0 & 0 & & & & 0 & 9 \\
\hline & 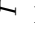 & POL & 18 & 4 & 7 & ? & 2 & 0 & 1 & 0 & 0 & 0 & 0 & 0 & 0 & 0 & 2 \\
\hline & & Avg & 15 & 14 & 11 & 5 & 3 & 2 & 1 & 0 & 0 & 0 & 0 & 0 & 0 & 0 & 4 \\
\hline & - & $\mathrm{ABI}$ & 100 & 99 & 97 & 92 & 92 & 92 & 96 & 94 & 8 & 94 & 88 & 78 & 77 & 74 & 90 \\
\hline & $\|$ & ATL & 99 & 86 & 86 & 76 & 70 & 64 & 54 & 0 & 5 & 4 & 43 & 4 & 4 & 60 & 63 \\
\hline & 5 & NoB & 88 & 91 & & 81 & 0 & 5 & 7 & - & 62 & 5 & & 5 & & 81 & 72 \\
\hline & -7 & POL & 98 & 75 & 82 & 75 & 67 & 64 & 57 & 56 & 0 & 66 & 64 & 11 & 68 & 60 & 69 \\
\hline & & Avg & 96 & 88 & 87 & 81 & 76 & 74 & 71 & 63 & 68 & 65 & 65 & 64 & 61 & 69 & 73 \\
\hline & $N$ & $\mathrm{ABI}$ & 100 & 100 & 100 & 99 & 100 & 10 & 100 & 100 & 100 & 100 & 100 & 100 & 100 & 100 & 100 \\
\hline & $\|$ & ATL & 100 & 99 & 97 & & 98 & 0 & 99 & 99 & 9 & 9 & 9 & 95 & 97 & 10 & 98 \\
\hline & & NoB & 100 & 100 & 99 & & 99 & & 98 & 9 & 9 & 10 & & 9 & $y$ & & 99 \\
\hline & -1 & POL & 99 & 98 & 99 & 99 & 97 & 100 & 97 & 100 & 91 & 98 & 98 & 96 & 94 & 98 & 97 \\
\hline & & Avg & 100 & 99 & 99 & 99 & 99 & 100 & 99 & 100 & 96 & 99 & 99 & 99 & 98 & 99 & 99 \\
\hline & $\infty$ & $\mathrm{ABI}$ & 10 & 100 & 10 & 10 & 10 & & 100 & 10 & 1 & 10 & & 100 & 10 & 10 & 100 \\
\hline & $\|$ & $\mathrm{AT}$ & & 10 & & & & & 10 & & & & & & & & 100 \\
\hline & $\pi$ & NoB & 100 & 10 & 100 & 10 & 100 & 100 & 100 & 100 & 10 & 10 & & 100 & 100 & 100 & 100 \\
\hline & - & POL & 100 & 100 & 100 & 100 & 100 & 100 & 100 & 100 & 100 & 100 & 100 & 100 & 100 & 99 & 100 \\
\hline & & Avg & 100 & 100 & 100 & 100 & 100 & 100 & 100 & 100 & 100 & 100 & 100 & 100 & 100 & 100 & 100 \\
\hline
\end{tabular}

function value (with a ratio close to 1.0 ), while it clearly outperforms the exact approaches on the harder instances $(|R| \geq 16)$ where, for $\Gamma=3$, the heuristic solutions are better by a factor larger than 3.5.

The results for the complete data set can be found in Table 4. Times are reported in Table 5. We remark that the heuristic method finds nonzero solutions for all the instances and for all values of $\Gamma$, whereas, with the exact method, we find nonzero solutions for only 87 cases out of 168 . For the cases where a solution to the exact formulation is known, we find solutions which are substantially better than the best ones found via the exact method within the time limit. On average, the heuristic methods yields solutions with an objective function value that is better by a factor of 1.42 . If we restrict to $\Gamma>0$, this factor goes up to 2.04 .

We remark that, for all the instances that can be solved to optimality with the exact approach, equivalent solution values are obtained with the heuristic approaches. This indicates that, at least on the smaller instances for which the optimal solution value is known, the heuristics are definitely competitive 
Table 5: Detailed computing times for the two-phase heuristic with $z_{L}=\left|V^{0}\right|, z_{M}=2$, and $z_{H}=1$

\begin{tabular}{|c|c|c|c|c|c|c|c|c|c|c|c|c|c|c|c|c|}
\hline & $|R|$ & 5 & 6 & 7 & 8 & 9 & 10 & 12 & 14 & 16 & 18 & 20 & 24 & 28 & 32 & Avg \\
\hline \multirow{15}{*}{ 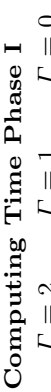 } & ABILENE & 0 & 0 & 0 & 0 & 1 & 5 & 6 & 7 & 1 & 2 & 2 & 2 & 1 & 4 & 2 \\
\hline & ATLANTA & 0 & 0 & 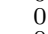 & 0 & 0 & 0 & 0 & 0 & 0 & 0 & 1 & 1 & 14 & 15 & 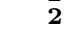 \\
\hline & NOBEL-US & 0 & 0 & 0 & 0 & 0 & 0 & 0 & 300 & 0 & 0 & 9 & 13 & 14 & 95 & $3 \overline{1}$ \\
\hline & POLSKA & 0 & 0 & 0 & 0 & 0 & 0 & 0 & 0 & 1 & 1 & 1 & 8 & 300 & 59 & 26 \\
\hline & Avg & 0 & 0 & 0 & $\mathbf{0}$ & $\mathbf{0}$ & 1 & 1 & 77 & 1 & 1 & 3 & 6 & 82 & 43 & \\
\hline & AbiLene & 0 & 0 & 0 & 0 & 0 & 0 & 0 & 0 & 0 & 0 & 0 & 1 & 300 & 300 & 43 \\
\hline & ATLANTA & 0 & 0 & 0 & 0 & 0 & 0 & 1 & 2 & 300 & 300 & 300 & 300 & 300 & 300 & 129 \\
\hline & NOBEL-US & 0 & 0 & 0 & 0 & & 1 & 1 & 8 & 300 & 300 & 300 & 300 & 300 & 300 & 129 \\
\hline & POLSKA & 0 & 0 & 0 & 0 & 0 & 0 & 0 & 300 & 300 & 300 & 300 & 300 & 300 & 300 & 150 \\
\hline & Avg & 0 & 0 & 0 & $\mathbf{0}$ & $\mathbf{0}$ & 0 & 1 & 77 & 225 & 225 & 225 & 225 & 300 & 300 & 113 \\
\hline & ABILENE & 0 & 0 & 0 & 1 & 0 & 1 & 0 & 1 & 13 & 13 & 22 & 47 & 300 & 272 & 48 \\
\hline & ATLANTA & 0 & 0 & & 1 & 2 & 3 & 300 & 300 & 300 & 300 & 300 & 300 & 300 & 300 & 172 \\
\hline & NOBEL-US & 0 & 0 & 0 & 300 & 300 & 300 & 300 & 300 & 300 & 300 & 300 & 300 & 300 & 300 & 236 \\
\hline & POLSKA & 0 & 1 & 300 & 300 & 300 & 300 & 300 & 300 & 300 & 300 & 300 & 300 & 300 & 300 & 257 \\
\hline & Avg & 0 & 0 & 75 & 150 & 151 & 151 & 225 & 225 & 228 & 228 & 230 & 237 & 300 & 293 & 178 \\
\hline \multirow{5}{*}{$\begin{array}{l}\infty \\
\| \\
L\end{array}$} & ABILENE & 0 & 0 & 0 & 1 & 0 & 1 & 1 & 1 & 2 & 2 & 2 & 34 & 68 & 80 & 14 \\
\hline & ATLANTA & 0 & 1 & 1 & 5 & 13 & 7 & 6 & 18 & 300 & 300 & 300 & 300 & 300 & 300 & 132 \\
\hline & NOBEL-US & 0 & 0 & 0 & 1 & 3 & 3 & 300 & 300 & 300 & 300 & 300 & 300 & 300 & 300 & 172 \\
\hline & POLSKA & 0 & 1 & 300 & 139 & 300 & 300 & 300 & 300 & 300 & 300 & 300 & 300 & 300 & 300 & 246 \\
\hline & Avg & 0 & 0 & 76 & 37 & 79 & 78 & 152 & 155 & 226 & 226 & 226 & 233 & 242 & 245 & 141 \\
\hline \multirow{20}{*}{ 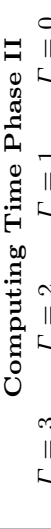 } & ABILENE & 0 & 0 & 0 & 0 & 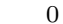 & 0 & 0 & 0 & 0 & 0 & 0 & 0 & 0 & 0 & 0 \\
\hline & ATLA & 0 & 0 & 0 & 0 & 0 & 0 & 1 & 2 & 3 & 9 & 5 & 13 & 107 & 17 & 11 \\
\hline & NOBEL-US & 0 & 0 & 0 & & 0 & 0 & 1 & 1 & 8 & 1 & 1 & 2 & 2 & 2 & 1 \\
\hline & POLSKA & 0 & 0 & 0 & 0 & 1 & 0 & 1 & 1 & 1 & 1 & 1 & 0 & 1 & 1 & 1 \\
\hline & Avg & 0 & 0 & $\mathbf{0}$ & & $\mathbf{0}$ & 0 & 1 & 1 & 3 & 3 & 2 & 4 & 28 & 5 & 3 \\
\hline & ABILE & 0 & 0 & 0 & 0 & 0 & 0 & 0 & 0 & 0 & 0 & 0 & 0 & 0 & 0 & 0 \\
\hline & ATLANTA & 0 & 0 & 0 & 0 & 0 & 0 & 0 & 0 & 0 & 0 & 0 & 9 & 0 & J & 1 \\
\hline & NOBEL-US & 0 & 0 & 0 & & 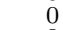 & 0 & 0 & 0 & 0 & 0 & 0 & 0 & 0 & 0 & 0 \\
\hline & POLSKA & 0 & 0 & 0 & & 0 & 0 & 0 & 0 & 0 & 0 & 0 & 1 & 0 & 0 & 0 \\
\hline & Avg & 0 & $\mathbf{0}$ & 0 & $\mathbf{0}$ & 0 & 0 & 0 & 0 & 0 & 0 & 0 & 2 & 0 & 0 & 0 \\
\hline & ABILF & 0 & 0 & 0 & 0 & 0 & 0 & 0 & 0 & 0 & 0 & 0 & 0 & 0 & 0 & 0 \\
\hline & ATLAI & 0 & 0 & 0 & &  & 0 & 0 & 0 & 0 & 0 & 0 & 0 & 0 & 0 & 0 \\
\hline & NOBEI & 0 & 0 & & & & ( & 0 & 0 & 0 & 0 & 0 & 0 & 0 & 0 & 0 \\
\hline & POLSKA & 0 & 0 & 0 & & 0 & 0 & 0 & 0 & 0 & 0 & 0 & 0 & 0 & 0 & 0 \\
\hline & Avg & 0 & 0 & $\mathbf{0}$ & 0 & 0 & 0 & 0 & 0 & $\mathbf{0}$ & 0 & 0 & 0 & 0 & 0 & 0 \\
\hline & ABILENE & 0 & 0 & 0 & 0 & 0 & 0 & 0 & 0 & 0 & 0 & 0 & 0 & 0 & 0 & 0 \\
\hline & ATLA & 0 & 0 & 0 & & 0 & 0 & 0 & 1 & 0 & 0 & 0 & 0 & 0 & 0 & 0 \\
\hline & NOBE & 0 & 0 & & 0 & 0 & 0 & 0 & 0 & 0 & 0 & 0 & 0 & 0 & 0 & 0 \\
\hline & POLSKA & 0 & 0 & & & 0 & 0 & 0 & 0 & 0 & 0 & 0 & 0 & 0 & 0 & 0 \\
\hline & Avg & 0 & $\mathbf{0}$ & 0 & 0 & $\mathbf{0}$ & 0 & 0 & $\mathbf{0}$ & $\mathbf{0}$ & $\mathbf{0}$ & 0 & 0 & $\mathbf{0}$ & 0 & $\mathbf{0}$ \\
\hline \multirow{10}{*}{ 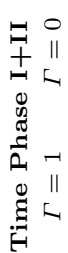 } & ABILENE & 0 & 0 & 0 & & 1 & 5 & 6 & 7 & 1 & 2 & 2 & 2 & 1 & 4 & 2 \\
\hline & ATLA & 0 & 0 & U & 0 & 0 & 0 & 1 & & 3 & $\overline{9}$ & 6 & 14 & 121 & 32 & $1 \overline{3}$ \\
\hline & NOBE & 0 & 0 & & & 0 & 0 & 1 & 301 & 9 & 1 & 10 & 15 & 16 & 97 & 32 \\
\hline & POLSK & 0 & 0 & & & 1 & & 1 & 1 & 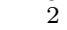 & 1 & 2 & 8 & 301 & 61 & 27 \\
\hline & Avg & 0 & 0 & 0 & 0 & 0 & 1 & 2 & 78 & 3 & 3 & 5 & 10 & 110 & 48 & 19 \\
\hline & ABILI & 0 & 0 & 0 & 0 & 0 & 0 & 0 & 0 & 0 & 0 & 0 & 1 & 300 & 300 & 3 \\
\hline & ATLAI & 0 & 0 & 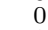 & 0 & 0 & 0 & 2 & 2 & 300 & 300 & 300 & 309 & 300 & 300 & 130 \\
\hline & NOBEI & 0 & 0 & & & & 1 & 1 & 8 & & 300 & 300 & 300 & & 300 & 129 \\
\hline & POLSK & 0 & 0 & 0 & & 1 & 1 & 1 & 300 & 300 & 300 & 300 & 301 & 300 & 300 & 150 \\
\hline & Avg & 0 & 0 & 0 & 0 & 0 & 0 & 1 & 78 & 225 & 225 & 225 & 228 & 300 & 300 & 113 \\
\hline \multirow{10}{*}{ 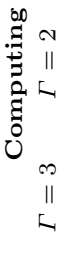 } & ABILE & 0 & 0 & & & & 1 & 0 & 1 & 1 & 3 & 22 & 47 & 30 & 272 & 48 \\
\hline & ATLAI & 0 & 0 & 0 & 1 & 3 & 4 & 300 & 300 & 300 & 300 & 300 & 300 & 300 & 300 & 172 \\
\hline & NoBEI & 0 & 0 & & 300 & 300 & & 300 & & & 300 & 300 & 300 & & 300 & 236 \\
\hline & POLSK & 0 & 1 & 300 & 300 & 300 & 300 & 300 & 300 & 300 & 300 & 300 & 300 & 300 & 300 & 257 \\
\hline & Avg & 0 & 0 & 75 & 151 & 151 & 151 & 225 & 225 & 228 & 228 & 231 & 237 & 300 & 293 & 178 \\
\hline & ABILEne & 0 & 0 & 0 & 1 & 0 & 1 & 1 & 1 & 2 & & 2 & 34 & 68 & 80 & 14 \\
\hline & ATLAI & 0 & 1 & 1 & 5 & 13 & 7 & 6 & 20 & 300 & 300 & 300 & 300 & 300 & 300 & 132 \\
\hline & NOBEL- & 0 & 0 & 0 & 1 & 4 & 3 & 300 & 300 & 300 & 300 & 300 & 300 & 300 & 300 & 172 \\
\hline & POLSKA & 0 & 1 & 300 & 139 & 300 & 300 & 300 & 300 & 300 & 300 & 300 & 300 & 300 & 300 & 246 \\
\hline & Avg & 0 & 0 & 76 & 37 & 79 & 78 & 152 & 155 & 226 & 226 & 226 & 234 & 242 & 245 & 141 \\
\hline
\end{tabular}


in terms of solution quality. Table 5 also illustrates how easier the second phase problem is w.r.t. the first one in terms of computing times.

It is worth mentioning though that, if one is not able to find a good parameter setting beforehand, the variability of the two-phase heuristic in terms of solution quality can be quite high. For a visual depiction, see Figure 7, which reports the solution values for all parameter settings for the ABILENE instance. This phenomenon better shown in Table 6 , which reports the minimum and maximum objective function value achieved with different parameter settings for each instance. Remarkably, the differences in solution quality are quite significant. As an example, for $\Gamma=2$ we have solutions between, on average, 427 and 261.

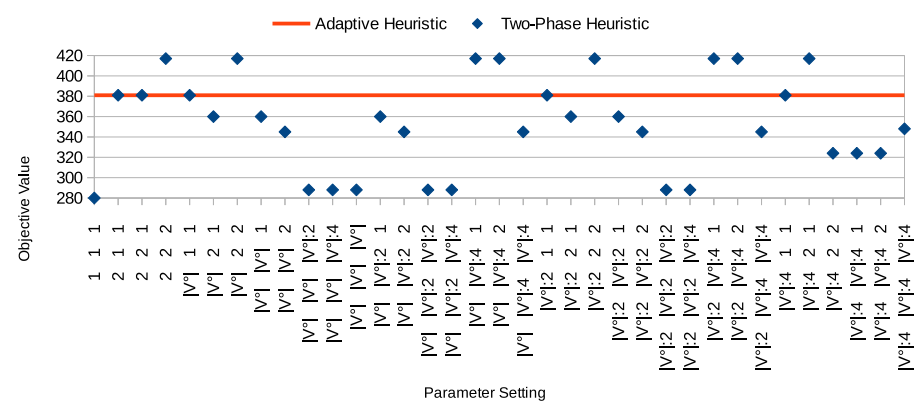

Fig. 7: Objective function values (blue) for the two-phase heuristic on the ABILENE instances, with $|R|=28$ and $\Gamma=1$ with $z_{L}, z_{M}, z_{H} \in\left\{\left|V^{0}\right|, \frac{\left|V^{0}\right|}{2}, \frac{\left|V^{0}\right|}{4}, 2,1\right\}$ and $z_{L} \leq z_{M} \leq z_{H}$. The corresponding solution obtained via the adaptive heuristic is shown in red.

Let us now consider the adaptive heuristic which, by design and differently from the two-phase heuristic, does not depend on a user-supplied parameter initialization. The corresponding results are shown in Table 7. As we can see, the adaptive heuristic provides results which are comparable to those obtained with the two-phase heuristic employing the "winning" parameter settingalthough, on average, the results for the former are slightly worse (although by a mere $3 \%$ ) than those for the latter. Nevertheless, there are cases where the adaptive heuristic improves over the two-phase heuristic with the "winning" parameter setting $z_{L}=\left|V^{0}\right|, z_{M}=2$, and $z_{H}=1$, such as for ABILENE with $|R|=28$, as can be observed in Figure 7 .

Overall, both heuristic methods dramatically outperform the exact approach, with the adaptive one being able to do so even without an a priori knowledge of a "good" parameter setting (although at the cost of a higher computing time when compared to the two-phase heuristic). 
Table 6: Best $(\max )$ and worst $(\min )$ solution values obtained via the two-phase heuristic over all parameter settings, i.e., $z_{L}, z_{M}, z_{H} \in\left\{\left|V^{0}\right|, \frac{\left|V^{0}\right|}{2}, \frac{\left|V^{0}\right|}{4}, 2,1\right\}$, with $z_{L} \leq z_{M} \leq z_{H}$. Entries are rounded to the nearest integer.

\begin{tabular}{|c|c|c|c|c|c|c|c|c|c|c|c|c|c|c|c|}
\hline$|R|$ & 5 & 6 & 7 & 8 & 9 & 10 & 12 & 14 & 16 & 18 & 20 & 24 & 28 & 32 & $\emptyset$ \\
\hline \multirow[t]{2}{*}{ ABILENE } & 34 & 381 & 438 & 471 & 500 & 500 & 512 & 512 & 95 & 632 & 632 & 632 & 664 & 715 & 538 \\
\hline & 2 & 301 & 339 & 304 & 332 & 332 & 304 & 318 & 342 & 379 & 438 & 396 & 413 & 369 & 342 \\
\hline & 402 & 444 & 523 & 559 & 623 & 695 & 853 & 970 & 1061 & 118 & 1206 & 1262 & 1416 & 1387 & 894 \\
\hline & 332 & 36 & 453 & 489 & 517 & 536 & 582 & 622 & 631 & 2 & 665 & 664 & 633 & 701 & 664 \\
\hline TOBEL- & 346 & 393 & 441 & 532 & 570 & 629 & 734 & 846 & 894 & 917 & 1011 & 1176 & 1223 & 1313 & 788 \\
\hline & 346 & 393 & 441 & 487 & 522 & 499 & 603 & 617 & 705 & 712 & 660 & 715 & 725 & 786 & 587 \\
\hline & 265 & 312 & 398 & 452 & 550 & 44 & 782 & 37 & 58 & 54 & 961 & 1011 & 1082 & 1141 & 747 \\
\hline & 265 & & 370 & 424 & 473 & 39 & 614 & 85 & 80 & 504 & 506 & 473 & 535 & 601 & 484 \\
\hline \multirow{2}{*}{$\begin{array}{l}\text { Avg (max) } \\
\text { Avg (min) }\end{array}$} & 339 & 83 & 450 & 504 & 61 & 617 & 720 & 791 & 880 & 930 & 953 & 1020 & 1096 & 1139 & 742 \\
\hline & 292 & 342 & 401 & 426 & 61 & 477 & 526 & 536 & 565 & 577 & 567 & 562 & 577 & 614 & 494 \\
\hline \multirow{2}{*}{ ABILENE } & 68 & 68 & 125 & 194 & 194 & 194 & 194 & 194 & 288 & 288 & 288 & 360 & 417 & 417 & 235 \\
\hline & 0 & 0 & 57 & 57 & 57 & 57 & 57 & 57 & 151 & 151 & 151 & 223 & 280 & 280 & 113 \\
\hline & 332 & 74 & 453 & 489 & 553 & 625 & 783 & 900 & 12 & 976 & 955 & 996 & 070 & 115 & 752 \\
\hline \| ATLA & 251 & 251 & 330 & 366 & 366 & 438 & 596 & 588 & 02 & 644 & 574 & 622 & 0 & 0 & 398 \\
\hline $\mathrm{NOF}$ & 294 & 341 & 389 & 480 & 518 & 518 & 623 & 652 & 31 & 636 & 649 & 744 & 776 & 775 & 573 \\
\hline & 19 & & 291 & 382 & 420 & 20 & 420 & 64 & 4 & 4 & 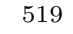 & 4 & 49 & 539 & 437 \\
\hline & 265 & 12 & 398 & 452 & 550 & 50 & 600 & 644 & 4 & 687 & 87 & 98 & 93 & 856 & 581 \\
\hline & 235 & 2 & 360 & 60 & 458 & 35 & 480 & 37 & & 532 & 30 & 25 & 01 & 574 & 455 \\
\hline \multirow{2}{*}{$\begin{array}{l}\text { Avg (max) } \\
\text { Avg (min) }\end{array}$} & 40 & 4 & 341 & 404 & 54 & 472 & 50 & 598 & & & 45 & 0 & 34 & 791 & 535 \\
\hline & 171 & 194 & 260 & 291 & 325 & 338 & 88 & 412 & 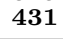 &  & 444 & 504 & 358 & 348 & 351 \\
\hline \multirow{2}{*}{ ABILENE } & 68 & 68 & 125 & 194 & 194 & 194 & 194 & 194 & 88 & 288 & 288 & 25 & 288 & 28 & 209 \\
\hline & 0 & 0 & 57 & 57 & 57 & 57 & 57 & 57 & 57 & 57 & 57 & 57 & 14 & 114 & 57 \\
\hline & 332 & 374 & 453 & 489 & 511 & 519 & 610 & 722 & 709 & 714 & 739 & 759 & 784 & 40 & 604 \\
\hline & 251 & 251 & 330 & 366 & 366 & 438 & 524 & 559 & 548 & 499 & 508 & 449 & 89 & 0 & 370 \\
\hline & 294 & 294 & 342 & 366 & 402 & 2 & 02 & 2 & & & & & 6 & & 402 \\
\hline $\mathrm{U}$ & & & 196 & 196 & 234 & 4 & 341 & 278 & 278 & 2 & 8 & 28 & 66 & 0 & 248 \\
\hline & 265 & 12 & 370 & 422 & 454 & 466 & 49 & 528 & 538 & 57 & 57 & 573 & $22-2$ & 704 & 493 \\
\hline POLSKA & 235 & 2 & 340 & 384 & 397 & 397 & 501 & 399 & 01 & 334 & (3) & 04 & 47 & 117 & 367 \\
\hline \multirow{2}{*}{$\begin{array}{l}\text { Avg (max) } \\
\text { Avg (min) }\end{array}$} & 240 & 262 & 323 & 368 & 90 & 395 & 425 & 462 & & 502 & 505 & 525 & 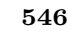 & 553 & 427 \\
\hline & & 182 & & 1 & 1 & 9 & 26 & 323 & & 2 & 01 & 10 & 4 & 133 & 261 \\
\hline \multirow{2}{*}{ ABILENE } & 68 & 68 & 125 & 137 & 137 & 137 & 137 & 137 & U & 220 & 220 & 220 & 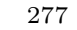 & 277 & 170 \\
\hline & 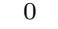 & & & & & 57 & 57 & 57 & 57 & 57 & 57 & 57 & & & 57 \\
\hline & 332 & 332 & 411 & 447 & 44 & 447 & 459 & 56 & 560 & 56 & 560 & 585 & 648 & 644 & 499 \\
\hline ATLANTA & 25 & a & 330 & 366 & 366 & 366 & 459 & 449 & 45 & 445 & 451 & 499 & 544 & 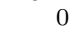 & 373 \\
\hline $\mathrm{NoB}$ & 225 & 225 & 27 & 28 & 32 & 320 & 32 & Jz & 3 & 3 & 227 & & & 40 & 331 \\
\hline US & ค & & 12 & 12 & 129 & 266 & 15 & 158 & 15 & 26 & 15 & 24 & 27 & 327 & 186 \\
\hline & 15 & 2 & 322 & 346 & 406 & 406 & 456 & 500 & $-5+3$ & 554 & 526 & 50 & 1 & 638 & 442 \\
\hline I OLSKA & 169 & & 0 & & $0 x$ & 271 & 321 & 305 & 305 & $32>$ & 324 & 56 & 99 & 428 & 300 \\
\hline \multirow{2}{*}{$\begin{array}{l}\text { Avg (max) } \\
\text { Avg (min) }\end{array}$} & 206 & & & 30 & 328 & 3 & & 379 & & & & & & 507 & 361 \\
\hline & 126 & 137 & 200 & 206 & 206 & 240 & 249 & 242 & 243 & 273 & 248 & 289 & 333 & 217 & 229 \\
\hline
\end{tabular}

5.4 Considerations on the protection level

We now focus on the empirical protection level achieved by the different approaches. Recall that, when adopting worst case or average data, the protection level is equal to 0 for the former whereas, as it can be observed computationally, it is almost always equal to $100 \%$ for the latter. 
Table 7: Results for the adaptive heursitic.

\begin{tabular}{|c|c|c|c|c|c|c|c|c|c|c|c|c|c|c|c|c|}
\hline & $|R|$ & 5 & 6 & 7 & 8 & 9 & 10 & 12 & 14 & 16 & 18 & 20 & 24 & 28 & 32 & Avg \\
\hline \multirow{20}{*}{ @ } & ABI & 322 & 381 & 438 & 450 & 480 & 480 & 472 & 472 & 566 & 507 & 507 & 564 & 513 & 658 & 486 \\
\hline & O ATL & 402 & 444 & 523 & 559 & 623 & 695 & 783 & 906 & 866 & 929 & 956 & 937 & 990 & 920 & 752 \\
\hline & $\|$ NoB & 346 & 393 & 441 & 532 & 570 & 629 & 734 & 770 & 879 & 842 & 836 & 1046 & 989 & 1106 & 722 \\
\hline & - POL & 265 & 312 & 398 & 452 & 550 & 644 & 752 & 739 & 750 & 740 & 597 & 626 & 724 & 798 & 596 \\
\hline & Avg & 334 & 383 & 450 & 498 & 556 & 612 & 685 & 722 & 765 & 755 & 724 & 793 & 804 & 871 & 639 \\
\hline & ABI & 68 & 68 & 125 & 194 & 194 & 194 & 194 & 194 & 288 & 288 & 288 & 360 & 381 & 381 & 230 \\
\hline & $\rightarrow$ ATL & 332 & 374 & 453 & 453 & 553 & 625 & 783 & 762 & 833 & 832 & 850 & 750 & 605 & 602 & 629 \\
\hline & $\|$ NoB & 294 & 341 & 389 & 480 & 480 & 473 & 586 & 607 & 636 & 630 & 609 & 728 & 750 & 748 & 554 \\
\hline & POL & 265 & 312 & 398 & 452 & 522 & 522 & 553 & 604 & 595 & 640 & 623 & 649 & 572 & 706 & 530 \\
\hline & Avg & 240 & 274 & $=341$ & 395 & 437 & 454 & 529 & 542 & 588 & 598 & 593 & 622 & 577 & 609 & 486 \\
\hline & ABI & 68 & 68 & 125 & 194 & 194 & 194 & 194 & 194 & 288 & 288 & 288 & 256 & 277 & 277 & 208 \\
\hline & v ATL & 332 & 374 & 453 & 447 & 511 & 519 & 610 & 686 & 722 & 660 & 662 & 659 & 552 & 550 & 553 \\
\hline & $\|$ NoB & 294 & 294 & 342 & 366 & 402 & 402 & 402 & 395 & 395 & 405 & 401 & 459 & 397 & 467 & 387 \\
\hline & POL & 265 & 284 & 368 & 394 & 454 & 454 & 478 & 475 & 491 & 498 & 492 & 548 & 583 & 606 & 456 \\
\hline & Avg & 240 & 255 & 322 & 350 & 390 & 392 & 421 & 438 & 474 & 463 & 461 & 481 & 452 & 475 & 401 \\
\hline & ABI & 68 & 68 & 125 & 126 & 126 & 126 & 126 & 126 & 220 & 220 & 220 & 220 & 220 & 220 & 158 \\
\hline & D ATL & 332 & 332 & 411 & 447 & 411 & 447 & 459 & 560 & 560 & 560 & 560 & 574 & 580 & 554 & 485 \\
\hline & $\|$ NoB & 225 & 225 & 260 & 282 & 320 & 320 & 320 & 312 & 320 & 320 & 337 & 420 & 419 & 456 & 324 \\
\hline & POL & 189 & 244 & 294 & 308 & 369 & 369 & 412 & 437 & 481 & 472 & 472 & 472 & 517 & 585 & 402 \\
\hline & Avg & 204 & 217 & 273 & 291 & 307 & 316 & 329 & 359 & 395 & 393 & 397 & 422 & 434 & 454 & 342 \\
\hline 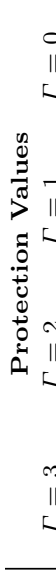 & ABI & 1 & 0 & 1 & 2 & 1 & 1 & 3 & 2 & 0 & 1 & 0 & 1 & 0 & 0 & 1 \\
\hline & D ATL & 75 & 72 & 75 & 1 & 1 & 0 & 2 & 0 & 0 & 0 & 1 & 0 & 0 & 0 & 16 \\
\hline & $\|$ NoB & 59 & 49 & 1 & 1 & 0 & 0 & 0 & 0 & 0 & 0 & 0 & 0 & 0 & 0 & 8 \\
\hline & POL & 96 & 86 & 23 & 2 & 3 & 0 & 0 & 1 & 0 & 0 & 1 & 1 & 0 & 0 & 15 \\
\hline & Avg & 58 & 52 & 25 & 2 & 1 & 0 & 1 & 1 & 0 & 0 & 1 & 1 & 0 & 0 & 10 \\
\hline & ABI & 98 & 98 & 98 & 97 & 99 & 99 & 99 & 94 & 89 & 94 & 87 & 71 & 62 & 80 & 90 \\
\hline & $\rightarrow$ ATL & 91 & 84 & 91 & 90 & 81 & 68 & 54 & 63 & 39 & 42 & 28 & 42 & 50 & 31 & 61 \\
\hline & $\|$ NoB & 88 & 84 & 81 & 82 & 75 & 85 & 60 & 56 & 42 & 52 & 54 & 57 & 49 & 61 & 66 \\
\hline & - POL & 90 & 81 & 75 & 72 & 68 & 68 & 59 & 66 & 69 & 61 & 62 & 59 & 63 & 57 & 68 \\
\hline & Avg & 92 & 87 & 86 & 85 & 81 & 80 & 68 & 70 & 60 & 62 & 58 & 58 & 56 & 57 & 71 \\
\hline & ABI & 100 & 100 & 100 & 100 & 100 & 100 & 100 & 100 & 98 & 98 & 98 & 99 & 100 & 100 & 100 \\
\hline & $N$ ATL & 99 & 98 & 99 & 100 & 99 & 99 & 99 & 96 & 96 & 100 & 98 & 98 & 98 & 99 & 99 \\
\hline & $\|$ NoB & 100 & 100 & 99 & 99 & 99 & 99 & 99 & 99 & 100 & 97 & 99 & 100 & 98 & 99 & 99 \\
\hline & POL & 99 & 99 & 97 & 95 & 100 & 100 & 95 & 98 & 95 & 96 & 99 & 98 & 94 & 96 & 97 \\
\hline & Avg & 100 & 99 & 99 & 99 & 100 & 100 & 98 & 98 & 97 & 98 & 99 & 99 & 98 & 99 & 99 \\
\hline & ABI & 100 & 100 & 100 & 100 & 100 & 100 & 100 & 100 & 100 & 100 & 100 & 100 & 99 & 99 & 00 \\
\hline & $\Rightarrow$ ATL & 100 & 00 & 100 & 100 & 100 & 10 & 1 & & 100 & 0 & & & 9 & 100 & 00 \\
\hline & $\|$ NoB & 100 & 100 & 100 & 100 & 100 & 100 & 100 & 100 & 100 & 100 & 100 & 100 & 100 & 100 & 100 \\
\hline & - POL & 100 & 100 & 100 & 100 & 100 & 100 & 100 & 100 & 99 & 100 & 100 & 100 & 100 & 99 & 100 \\
\hline & Avg & 100 & 100 & 100 & 100 & 100 & 100 & 100 & 100 & 100 & 100 & 100 & 100 & 100 & 100 & 100 \\
\hline \multirow{15}{*}{ - } & BI & 6 & 14 & 12 & 37 & 29 & 3 & 13 & 34 & 42 & 15 & 49 & 6 & 62 & 67 & 36 \\
\hline & D AT & 9 & 9 & 11 & 36 & 59 & 67 & 99 & 207 & 267 & 334 & 3 & 3 & 464 & 464 & 195 \\
\hline & $\|$ NoB & 8 & 10 & 8 & 8 & 8 & 9 & 44 & 128 & 137 & 211 & 206 & 465 & 486 & 614 & 167 \\
\hline & $-\mathrm{POL}$ & 6 & 7 & 16 & 12 & 27 & 51 & 125 & 97 & 110 & 116 & 90 & 97 & 94 & 119 & 69 \\
\hline & Avg & 7 & 10 & 12 & 23 & 31 & 40 & 70 & 116 & 139 & 177 & 174 & 244 & 276 & 316 & 117 \\
\hline & ABI & 6 & 6 & 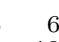 & 8 & 7 & 8 & 8 & 9 & 7 & 7 & 9 & 41 & 38 & 36 & 14 \\
\hline & - ATL & 11 & 0 & 10 & 10 & 32 & 17 & 127 & 180 & 522 & 2059 & 3180 & 3484 & 623 & 1231 & 821 \\
\hline & $\|$ NoB & 8 & 9 & 9 & 9 & 8 & 11 & 13 & 45 & 310 & 309 & 611 & 613 & 1518 & 613 & 292 \\
\hline & $-\mathrm{POL}$ & 6 & 6 & 12 & 30 & 34 & 62 & 68 & 213 & 308 & 551 & 569 & 2798 & 1891 & 1973 & 609 \\
\hline & Avg & 8 & 7 & 9 & 14 & 20 & 24 & 54 & 112 & 287 & 732 & 1093 & 1734 & 1017 & 963 & 434 \\
\hline & $\mathrm{BI}$ & 7 & 11 & 9 & 15 & 10 & 11 & 1 & 1 & 1 & 33 & 1 & 1 & 912 & 910 & 20 \\
\hline & N ATL & 10 & 9 & 10 & 10 & 29 & 18 & 1664 & 3098 & 3445 & 2137 & 3042 & 3361 & 622 & 613 & 1291 \\
\hline & $\|$ NoB & 8 & 8 & 8 & 308 & 308 & 309 & 309 & 308 & 309 & 311 & 308 & 308 & 308 & 308 & 244 \\
\hline & $-\mathrm{P}$ & 6 & 6 & 6 & 367 & 307 & 307 & 2114 & 3320 & 3318 & 3357 & 3600 & 2716 & 3466 & 3320 & 1872 \\
\hline & Avg & 8 & 9 & 8 & 175 & 164 & 161 & 1024 & 1684 & 1776 & 1459 & 1745 & 1824 & 1327 & 1288 & 904 \\
\hline & ABI & 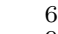 & 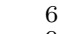 & 6 & 306 & 307 & 307 & 307 & 308 & 促 & 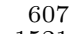 & 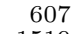 & 607 & 08 & 1209 & 500 \\
\hline$\infty$ & $\Rightarrow$ ATL & 9 & 9 & 10 & 10 & 10 & 15 & 15 & 21 & 1222 & 1521 & 1519 & 1819 & 2136 & 1820 & 724 \\
\hline$\|$ & $\|$ NoB & 8 & 8 & 8 & 8 & 8 & 8 & 307 & 308 & 308 & 308 & 308 & 612 & 1214 & 1211 & 330 \\
\hline L & $-\mathrm{POL}$ & 6 & 6 & 308 & 607 & 2173 & 1528 & 2020 & 3019 & 2819 & 3316 & 3314 & 3315 & 3389 & 2717 & 2039 \\
\hline & Avg & 7 & 7 & 83 & 233 & 625 & 465 & 662 & 914 & 1240 & 1438 & 1437 & 1588 & 2137 & 1739 & 898 \\
\hline
\end{tabular}


Let us first consider the exact $\Gamma$-robust approach for $\Gamma \in\{1,2,3\}$, as reported in Table 2. We remark that the empirical protection level is induced by the feasibility of a solution and not by its optimality. Therefore, it is reasonable to measure the former for all the solutions provided by the method, regardless of them being optimal or not. Quite interestingly, we observe that, although the empirical protection level for $\Gamma=1$ is not very high (being equal to, on average, $82 \%$ ), it already reaches a value of, on average, $100 \%$ for $\Gamma=2$.

Similar observations can be drawn with both of our heuristics, as reported in Table 4 and in Table 7. For $\Gamma=0$ (the case with average data), the empirical protection level is, as expected, very small (3\% on average for the two-phase heuristic and $10 \%$ for the adaptive one). For $\Gamma=1$, it is still not very high for both methods, being close to $71 \%$ on average for both. Differently, for $\Gamma=2,3$ we obtain solutions with very few violations and a higher empirical protection level equal to, respectively, $99 \%$ and $100 \%$ on average (again for both heuristics).

\subsection{Recommendations}

As a consequence of the results that we observed in our experiments, we would advise to resort to the exact MILP formulation for the nominal case of VNE only with no more than $|R|=20$ requests and to the exact $\Gamma$-robust formulation only for $\Gamma=1$ and with up to $|R|=10$ requests. For all the other cases, we would suggest the adoption of our two-phase heuristic (which, among the two proposed algorithms, is definitely the faster one) with parameters $z_{L}=\left|V^{0}\right|, z_{M}=2$, and $z_{H}=1$. In case substantial differences in solution quality can be observed by experimenting with other parameter values (such differences could be substantial, as illustrated in Table 6) and a fine parameter tuning cannot be carried out in a preprocessing step, we advise the adoption of the computationally more demanding but, without a good guess on a suitable parameter choice, more stable, adaptive heuristic.

As to the choice of $\Gamma$, when aiming for a very high, i.e., $>95 \%$, protection level, we would advise, based on our experiments, to select $\Gamma=2$ for the node capacity constraints, while (possibly) letting $\Gamma=0$ for the link capacity ones.

\subsection{Results on larger instances}

To better assess how our algorithms scale on instances of larger size, we conclude the section by reporting on a set of experiments carried out on 8 physical networks taken from the Internet Topology Zoo database [24], Fatman, Digex, Cernet, Bellsouth, Intellifiber, RedBestel, Deltacom, and Cogentco, with, resp., $\left|V^{0}\right|=20,30,40,60,72,83,112$, and 199. Due to their size (larger than those used in the previous experiments), we consider up to $|R|=50 \mathrm{VN}$ requests.

We experiment with our two-phase heuristic, adopting the parameter setting $z_{L}=\left|V^{0}\right|, z_{M}=2, z_{H}=1$ which we have found to perform better in the 
previous experiments, with $\Gamma=1$ in phase one and $\Gamma=0$ in phase two. The results are illustrated in Table 8 . Note that, in the table, the computing time accounts for the total time spent in the two phases, neglecting the preprocessing time invested to compute the all pairs shortest paths which are needed for the distance-bounding constraints in phase one.

Overall, the table shows that our two-phase algorithm can solve reasonably well VNE instances with large physical networks (Cogentco contains $\left|V^{0}\right|=$ 199 nodes) with up to $|R|=40$ simultaneous VN requests, while the method starts to fail for $|R|=45,50$ due to the solver failing to find a nonzero solution to the phase one subproblem in the time limit.

The table also illustrates an interesting phenomenon. Indeed, it clearly shows that, although both subproblems get harder, as one would expect, when $|R|$ increases, the phase one subproblem gets substantially easier when the size of the physical network $\left|V^{0}\right|$ grows. This is, most likely, a feature of the underlying multi-knapsack structure, due to which the introduction of more physical nodes only makes node capacity a more abundant resource, without complicating the structure of the problem too much. Interestingly, the situation is reversed for the phase two subproblem, which gets harder for physical networks of larger size. This is, possibly, a consequence of the network topologies still playing a large role in it, so that, having a physical network of increased size does not directly translate into a problem which is easier to solve.

We remark that these two opposite behaviors are, quite interestingly, somewhat complementary in that, by increasing the value of $\left|V^{0}\right|$, while the phase one subproblem gets harder, the phase two subproblem gets easier. Overall, we end up with a situation where, if one of the two subproblems is not solved to optimality, then the other one (in most of the cases) is, thus obtaining an, overall, still effective algorithm.

\section{Concluding remarks}

Based on a chance-constrained formulation for the Virtual Network Embedding problem where node and traffic demands of the virtual networks are assumed to be random variables, we have proposed an exact $\Gamma$-robust MixedInteger Linear Programming (MILP) formulation which allows to find solutions with large profits that are guaranteed to be feasible with a high probability. Based on this formulation, which is suitable to solve small size instances in a reasonable amount of computing time but which scales poorly for larger networks, we have introduced two MILP-based $\Gamma$-robust heuristics: a twophase heuristic and an adaptive one.

Computational experiments indicate that, while the exact approaches become computationally challenging for instances with an increasing number of virtual network requests, both heuristics provide high quality solutions even for larger problems. We advise to adopt the first heuristic for the case where its input parameters can be determined beforehand whereas, if this is not the 
Table 8: Results on 8 Internet Topology Zoo instances of increasing size $\left|V^{0}\right|$. Entries are rounded to the nearest integer.

\begin{tabular}{|c|c|c|c|c|c|c|c|c|c|c|c|c|c|c|c|c|c|c|}
\hline & $\mid V^{0}$ & $R \mid$ & 8 & 9 & 10 & 12 & 14 & 16 & 18 & 20 & 24 & 28 & 32 & 35 & 40 & 45 & 50 & Avg \\
\hline 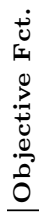 & $\begin{array}{r}20 \\
30 \\
40 \\
50 \\
72 \\
83 \\
112 \\
199\end{array}$ & 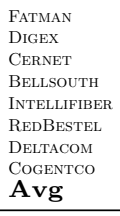 & $\begin{array}{r}328 \\
405 \\
396 \\
366 \\
385 \\
280 \\
343 \\
365 \\
\mathbf{3 5 9} \\
\end{array}$ & $\begin{array}{l}302 \\
409 \\
477 \\
379 \\
428 \\
379 \\
408 \\
459 \\
\mathbf{4 0 5} \\
\end{array}$ & $\begin{array}{l}331 \\
439 \\
477 \\
478 \\
442 \\
349 \\
472 \\
496 \\
\mathbf{4 3 6} \\
\end{array}$ & $\begin{array}{l}315 \\
422 \\
664 \\
459 \\
441 \\
380 \\
541 \\
534 \\
\mathbf{4 7 0} \\
\end{array}$ & $\begin{array}{r}341 \\
422 \\
679 \\
497 \\
538 \\
407 \\
687 \\
631 \\
\mathbf{5 2 5} \\
\end{array}$ & $\begin{array}{l}450 \\
424 \\
820 \\
544 \\
493 \\
416 \\
547 \\
566 \\
\mathbf{5 3 3} \\
\end{array}$ & $\begin{array}{l}417 \\
487 \\
704 \\
582 \\
496 \\
443 \\
619 \\
684 \\
\mathbf{5 5 4} \\
\end{array}$ & $\begin{array}{l}429 \\
476 \\
692 \\
734 \\
579 \\
458 \\
632 \\
740 \\
\mathbf{5 9 3} \\
\end{array}$ & $\begin{array}{l}370 \\
450 \\
724 \\
571 \\
583 \\
505 \\
806 \\
702 \\
\mathbf{5 8 9} \\
\end{array}$ & $\begin{array}{l}413 \\
445 \\
811 \\
763 \\
620 \\
543 \\
803 \\
778 \\
\mathbf{6 4 7} \\
\end{array}$ & $\begin{array}{l}523 \\
422 \\
724 \\
827 \\
524 \\
565 \\
695 \\
793 \\
\mathbf{6 3 4} \\
\end{array}$ & $\begin{array}{l}468 \\
235 \\
911 \\
789 \\
576 \\
522 \\
617 \\
779 \\
\mathbf{6 1 2} \\
\end{array}$ & $\begin{array}{l}434 \\
284 \\
682 \\
864 \\
599 \\
540 \\
833 \\
837 \\
\mathbf{6 3 4} \\
\end{array}$ & $\begin{array}{r}441 \\
305 \\
0 \\
758 \\
0 \\
0 \\
996 \\
0 \\
313 \\
\end{array}$ & $\begin{array}{r}391 \\
0 \\
0 \\
0 \\
290 \\
509 \\
0 \\
0 \\
\mathbf{1 4 9} \\
\end{array}$ & $\begin{array}{l}397 \\
375 \\
584 \\
574 \\
466 \\
420 \\
600 \\
558 \\
497 \\
\end{array}$ \\
\hline 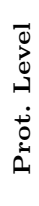 & $\begin{array}{r}20 \\
30 \\
40 \\
50 \\
72 \\
83 \\
112 \\
199\end{array}$ & 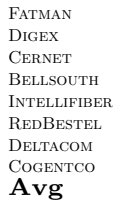 & $\begin{array}{r}99 \\
100 \\
97 \\
100 \\
100 \\
100 \\
98 \\
100 \\
99\end{array}$ & $\begin{array}{r}100 \\
100 \\
100 \\
99 \\
99 \\
100 \\
99 \\
99 \\
\mathbf{1 0 0}\end{array}$ & $\begin{array}{r}98 \\
100 \\
100 \\
100 \\
99 \\
99 \\
99 \\
100 \\
99\end{array}$ & $\begin{array}{r}98 \\
100 \\
100 \\
99 \\
99 \\
98 \\
99 \\
98 \\
99\end{array}$ & $\begin{array}{r}95 \\
99 \\
100 \\
97 \\
99 \\
99 \\
98 \\
100 \\
\mathbf{9 8}\end{array}$ & $\begin{array}{r}92 \\
98 \\
97 \\
100 \\
99 \\
99 \\
99 \\
100 \\
98\end{array}$ & $\begin{array}{r}98 \\
93 \\
92 \\
98 \\
99 \\
100 \\
99 \\
100 \\
\mathbf{9 7}\end{array}$ & $\begin{array}{r}96 \\
97 \\
90 \\
96 \\
93 \\
97 \\
98 \\
100 \\
\mathbf{9 6}\end{array}$ & $\begin{array}{l}91 \\
93 \\
96 \\
95 \\
98 \\
97 \\
97 \\
99 \\
\mathbf{9 6}\end{array}$ & $\begin{array}{l}97 \\
92 \\
99 \\
88 \\
96 \\
95 \\
98 \\
99 \\
96\end{array}$ & $\begin{array}{r}94 \\
100 \\
94 \\
91 \\
84 \\
93 \\
97 \\
99 \\
\mathbf{9 4}\end{array}$ & $\begin{array}{r}96 \\
100 \\
90 \\
95 \\
93 \\
97 \\
95 \\
98 \\
\mathbf{9 6}\end{array}$ & $\begin{array}{r}94 \\
100 \\
92 \\
92 \\
98 \\
85 \\
95 \\
95 \\
94\end{array}$ & $\begin{array}{r}90 \\
100 \\
100 \\
98 \\
100 \\
100 \\
95 \\
99 \\
98\end{array}$ & $\begin{array}{r}97 \\
100 \\
100 \\
100 \\
100 \\
99 \\
93 \\
97 \\
\mathbf{9 8}\end{array}$ & $\begin{array}{l}96 \\
98 \\
96 \\
97 \\
97 \\
97 \\
97 \\
99 \\
97\end{array}$ \\
\hline $\begin{array}{l}\text { के } \\
\text { कै } \\
\dot{0}\end{array}$ & $\begin{array}{r}20 \\
30 \\
40 \\
50 \\
72 \\
83 \\
112 \\
199\end{array}$ & 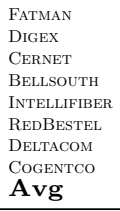 & $\begin{array}{l}0 \\
0 \\
0 \\
\mathbf{0}\end{array}$ & $\begin{array}{l}0 \\
0 \\
0 \\
\mathbf{0}\end{array}$ & 0 & $\begin{array}{l}0 \\
0 \\
0 \\
\mathbf{0}\end{array}$ & $\begin{array}{l}U \\
0\end{array}$ & $\begin{array}{l}0 \\
0 \\
0 \\
\mathbf{0}\end{array}$ & $\begin{array}{l}0 \\
0 \\
0 \\
\mathbf{2}\end{array}$ & $\begin{array}{l}0 \\
0 \\
0 \\
\mathbf{4}\end{array}$ & $\begin{array}{r}17 \\
0\end{array}$ & $\begin{array}{r}50 \\
64 \\
38 \\
0 \\
0 \\
0 \\
0 \\
0 \\
0 \\
\mathbf{1 9}\end{array}$ & $\begin{array}{r}44 \\
184 \\
53 \\
5\end{array}$ & $\begin{array}{r}48 \\
821 \\
50\end{array}$ & $\begin{array}{r}78 \\
639 \\
68 \\
8 \\
18\end{array}$ & $\begin{array}{r}78 \\
472 \\
\infty \\
71 \\
\infty \\
\infty \\
0 \\
0 \\
0 \\
\mathbf{1 2 4}\end{array}$ & $\begin{array}{r}78 \\
\infty \\
\infty \\
\infty \\
684 \\
78 \\
2 \\
0 \\
168 \\
\end{array}$ & $\begin{array}{r}29 \\
158 \\
18 \\
6 \\
51 \\
6 \\
0 \\
0 \\
33\end{array}$ \\
\hline $\begin{array}{l}\text { के } \\
\text { एँ } \\
\dot{0}\end{array}$ & $\begin{array}{r}20 \\
30 \\
40 \\
50 \\
72 \\
83 \\
112 \\
199\end{array}$ & 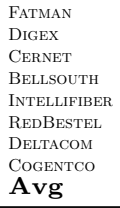 & 0 & $\begin{array}{l}0 \\
0 \\
0 \\
0 \\
0 \\
\mathbf{0}\end{array}$ & 0 & $\begin{array}{l}0 \\
\mathbf{0}\end{array}$ & 0 & $\begin{array}{l}0 \\
0 \\
0 \\
0 \\
5 \\
\mathbf{1}\end{array}$ & $\begin{array}{l}0 \\
0 \\
\mathbf{0}\end{array}$ & 0 & $\begin{array}{r}0 \\
0 \\
0 \\
0 \\
10 \\
\mathbf{1}\end{array}$ & $\begin{array}{l}8 \\
8 \\
\mathbf{3}\end{array}$ & 2 & $\begin{array}{r}0 \\
0 \\
11 \\
0 \\
11 \\
\mathbf{3}\end{array}$ & $\begin{array}{r}11 \\
\mathbf{3}\end{array}$ & $\begin{array}{r}0 \\
0 \\
0 \\
5 \\
\infty \\
1\end{array}$ & $\begin{array}{l}0 \\
0 \\
0 \\
0 \\
\infty \\
\infty \\
\mathbf{0}\end{array}$ & $\begin{array}{l}0 \\
0 \\
0 \\
1 \\
1 \\
1 \\
2 \\
4 \\
1\end{array}$ \\
\hline है & $\begin{array}{r}20 \\
30 \\
40 \\
50 \\
72 \\
83 \\
112 \\
199\end{array}$ & $\begin{array}{l}\text { Digex } \\
\text { CERnEt } \\
\text { BELLSOUth } \\
\text { INTELLIFIBER } \\
\text { REDBESTEL } \\
\text { DeLTACom } \\
\text { Cogentco } \\
\text { Avg }\end{array}$ & $\begin{array}{l}1 \\
8 \\
6 \\
8\end{array}$ & $\begin{array}{r}1 \\
6 \\
2 \\
1 \\
9 \\
6 \\
15 \\
84 \\
\mathbf{1 6}\end{array}$ & $\begin{array}{r}10 \\
3 \\
2 \\
1 \\
10 \\
20 \\
18 \\
51 \\
\mathbf{1 4}\end{array}$ & $\begin{array}{r}3 \\
28 \\
12 \\
42 \\
78 \\
\mathbf{5 9}\end{array}$ & $\begin{array}{r}300 \\
11 \\
6 \\
5 \\
21 \\
33 \\
78 \\
160 \\
77\end{array}$ & $\begin{array}{r}301 \\
30 \\
9 \\
4 \\
44 \\
56 \\
118 \\
304 \\
108\end{array}$ & $\begin{array}{r}301 \\
328 \\
72 \\
7 \\
107 \\
86 \\
115 \\
84 \\
\mathbf{1 3 7}\end{array}$ & $\begin{array}{r}338 \\
315 \\
9 \\
66 \\
114 \\
96 \\
164 \\
\mathbf{1 7 5}\end{array}$ & $\begin{array}{l}318 \\
140 \\
124 \\
236 \\
163 \\
307 \\
\mathbf{2 3 8}\end{array}$ & $\begin{array}{l}330 \\
327 \\
256 \\
265 \\
333 \\
309 \\
304 \\
\mathbf{3 0 3}\end{array}$ & $\begin{array}{r}301 \\
307 \\
321 \\
373 \\
600 \\
181 \\
312 \\
310 \\
\mathbf{3 3 8}\end{array}$ & $\begin{array}{l}300 \\
326 \\
331 \\
458 \\
600 \\
295 \\
311 \\
\mathbf{3 6 5}\end{array}$ & $\begin{array}{r}327 \\
372 \\
404 \\
600 \\
359 \\
321 \\
\mathbf{3 7 3}\end{array}$ & $\begin{array}{r}299 \\
315 \\
0 \\
0 \\
553 \\
342 \\
\mathbf{1 8 9}\end{array}$ & $\begin{array}{r}299 \\
299 \\
3 \\
202 \\
600 \\
313 \\
\mathbf{2 1 5}\end{array}$ & $\begin{array}{l}201 \\
152 \\
175 \\
141 \\
143 \\
166 \\
205 \\
210 \\
174\end{array}$ \\
\hline
\end{tabular}

case, we suggest to employ the adaptive heuristic, which provides competitive solutions, albeit at the cost of a larger investment in computing time. Experimenting with different values of $\Gamma$, so to establish a trade-off between the objective function value and the probability of being feasible for all realizations of the uncertain data, we have observed that, in our setting, $\Gamma=2$ provides the most favorable option, yielding at the same time solutions with a high objective function value which are, empirically, feasible with a very high probability.

Acknowledgements This work is supported by the

This work is supported by the BMWi grant 03ET7528B.

German Federal Ministry of Education and Research (BMBF grant 05M13PAA, joint project 05M2013 - VINO: Virtual Network Optimization). 


\section{References}

1. S. Coniglio, A. Koster, and M. Tieves, "Virtual network embedding under uncertainty: Exact and heuristic approaches," in Design of Reliable Communication Networks (DRCN), 2015 11th International Conference on the, pp. 1-8, 2015.

2. N. Chowdhury and R. Boutaba, "A survey of network virtualization," Computer Networks, vol. 54, no. 5, pp. 862-876, 2010.

3. A. Fischer, J. Botero, M. Beck, H. D. Meer, and X. Hesselbach, "Virtual network embedding: A survey," Communications Surveys \&S Tutorials, IEEE, vol. 15, no. 4, 2013.

4. N. Chowdhury and R. Boutaba, "Network virtualization: state of the art and research challenges," Communications Magazine, IEEE, vol. 47, pp. 20-26, July 2009.

5. M. Yu, Y. Yi, J. Rexford, and M. Chiang, "Rethinking virtual network embedding: substrate support for path splitting and migration," ACM SIGCOMM Computer Communication Review, vol. 38, no. 2, pp. 17-29, 2008.

6. E. Amaldi, S. Coniglio, A. Koster, and M. Tieves, "On the computational complexity of the virtual network embedding problem," Electronic Notes Discrete Mathematics, 2015. To appear.

7. Y. Zhu and M. Ammar, "Algorithms for assigning substrate network resources to virtual network components," in INFOCOM 2006, IEEE, pp. 1-12, IEEE, 2006.

8. M. Chowdhury, M. R. Rahman, and R. Boutaba, "Vineyard: Virtual network embedding algorithms with coordinated node and link mapping," IEEE/ACM Transactions on Networking (TON), vol. 20, no. 1, pp. 206-219, 2012.

9. A. Jarray and A. Karmouch, "Decomposition approaches for virtual network embedding with one-shot node and link mapping," Networking, IEEE/ACM Transactions on, vol. 23, pp. 1012-1025, June 2015.

10. X. Cheng, S. Su, Z. Zhang, H. Wang, F. Yang, Y. Luo, and J. Wang, "Virtual network embedding through topology-aware node ranking," SIGCOMM Computer Communication Reviews, vol. 41, pp. 38-47, Apr. 2011.

11. Z. Zhang, S. Su, J. Zhang, K. Shuang, and P. Xu, "Energy aware virtual network embedding with dynamic demands: Online and offline," Computer Networks, vol. 93, pp. 448-459, 2015.

12. I. Houidi, W. Louati, W. Ben-Ameur, and D. Zeghlache, "Virtual network provisioning across multiple substrate networks," Computer Networks, vol. 55, no. 4, pp. 1011-1023, 2011.

13. S. Coniglio, B. Grimm, A. Koster, M. Tieves, and A. Werner, "Optimal offline virtual network embedding with rent-at-bulk aspects," arXiv preprint, 2015. arXiv:1501.07887.

14. J. F. Botero, X. Hesselbach, M. Duelli, D. Schlosser, A. Fischer, and H. De Meer, "Energy efficient virtual network embedding," IEEE Commununication Letters, vol. 16 , no. 5, pp. 756-759, 2012.

15. I. Houidi, W. Louati, and D. Zeghlache, "Exact multi-objective virtual network embedding in cloud environments," The Computer Journal, vol. 58, no. 3, pp. 403-415, 2015.

16. M. Mangili, F. Martignon, and A. Capone, "Stochastic planning for content delivery: Unveiling the benefits of network functions virtualization," in Network Protocols (ICNP), 2014 IEEE 22nd International Conference on, pp. 344-349, Oct 2014.

17. J. Elias, F. Martignon, S. Paris, and J. Wang, "Optimization models for congestion mitigation in virtual networks," in Network Protocols (ICNP), 2014 IEEE 22nd International Conference on, pp. 471-476, Oct 2014.

18. G. Sun, H. Yu, L. Li, V. Anand, Y. Cai, and H. Di, "Exploring online virtual networks mapping with stochastic bandwidth demand in multi-datacenter," Photonic Network Communications, vol. 23, no. 2, pp. 109-122, 2012.

19. H. Kellerer, U. Pferschy, and D. Pisinger, "Introduction to NP-Completeness of Knapsack Problems," in Knapsack Problems, pp. 483-493, Springer Berlin Heidelberg, 2004.

20. A. Koster, M. Kutschka, and C. Raack, "Robust network design: formulations, valid inequalities, and computations," Networks, vol. 61, no. 2, pp. 128-149, 2013.

21. D. Bertsimas and M. Sim, "Robust discrete optimization and network flows," in Math. Prog., vol. 98, pp. $49-71,2003$. 
22. D. Bertsimas and M. Sim, "The price of robustness," Operations Research, vol. 52, no. 1, pp. $35-53,2004$

23. "SNDlib." http://sndlib.zib.de.

24. S. Knight, H. Nguyen, N. Falkner, R. Bowden, and M. Roughan, "The internet topology zoo," Selected Areas in Communications, IEEE Journal on, vol. 29, pp. 1765-1775, October 2011

Stefano Coniglio is a Lecturer in Operational Research at the University of Southampton. He received his Ph.D. in Information Technology in 2011 from Politecnico di Milano, Italy. His research focuses on mathematical programming and combinatorial optimization.

Arie M.C.A. Koster is a Full Professor of Discrete Optimization at RWTH Aachen University, Germany. He received his Ph.D. degree in Mathematics of Operations Research (1999) from Maastricht University, The Netherlands. His research interests are in the development and application of discrete optimization methods, including algorithmic graph theory and robust optimization.

Martin Tieves is a Ph.D. student in Mathematics at RWTH Aachen University, Germany. He holds a Master of Science in Mathematics (minor: Business Administration) from the same university (2011). He is interested in the optimization of robust and dynamic communication networks. 